\title{
Dynamics and variability of neuronal subtype responses during regeneration and
} growth

Jamie A. Havrilak ${ }^{1}$, Layla Al-Shaer ${ }^{1}$, Noor Baban ${ }^{1}$, Nesli Akinci ${ }^{1}$, Michael J. Layden ${ }^{*}$

${ }^{1}$ Lehigh University, Department of Biological Sciences, Bethlehem, PA

*Corresponding Author: layden@lehigh.edu

Keywords: Nematostella, regeneration, neurogenesis, nerve net, LWamide, regenerative neurogenesis, nervous system scalability 


\begin{abstract}
:
Background: We are interested in how nervous systems regenerate during whole-body axis regeneration. During whole-body axis regeneration, lost tissues are replaced to restore normal adult morphology. Typically, regenerates are smaller than their preamputated parent and have normal proportionality, suggesting that tissue scaling occurs in regenerates. The cell dynamics and responses of neuronal subtypes during nervous system regeneration in animals undergoing whole-body axis regeneration are not well understood in any system. The cnidarian sea anemone Nematostella vectensis is capable of whole-body axis regeneration, and its transparent, "simple" body plan, and the availability of fluorescent reporter lines, allows for the ability and resolution to track neural subtypes throughout the entire regenerative process. We utilized the $N v L W a m i d e-$ like::mCherry neuronal subtype transgenic reporter line to determine the in vivo response of neuronal subtypes during Nematostella vectensis nerve net regeneration and scaling.
\end{abstract}

Results: Regenerates were smaller than their pre-amputated size, and the regenerated nerve nets were reduced in neuronal number. Different neuronal subtypes had several distinct responses that included consistent, no, and conditional regeneration. Conditional regeneration relied in part on the size of the remnant fragment. Regenerates showed normal behaviors, indicating that the reduced nerve net retains functionality. These observations led us to investigate nerve net scaling. Neuronal numbers decreased as animals shrunk, and increased as they grew, and the changes were reversible. As with regenerates, behaviors present prior to the nervous system adjusting in size were also present after, regardless of whether the number of neurons increased or decreased.

Conclusion: These data suggest that the Nematostella nerve net is dynamic, capable of scaling with changes in body size, and that neuronal subtypes display differential regenerative capabilities, which is likely linked to the scale-state of the regenerating nervous system. 


\section{Introduction:}

We are interested in understanding the mechanisms that regulate how nervous systems are reformed during whole-body axis regeneration. While investigation of hydrozoan cnidarian and planarian regeneration has provided insights regarding general molecular mechanisms that promote regenerative neurogenesis [1-9], how individual neuronal fates respond and reform during regeneration remains poorly understood.

During whole-body axis regeneration animals completely regenerate entire portions of their body. However, regenerates are typically smaller than the pre-amputated animal. In planarians, the smaller regenerates display correct proportionality, suggesting regenerated and preexisting tissues are repatterned to scale [10-13]. Planarians grow and shrink in response to feeding and starvation, and neuronal number positively correlates with length, which suggests that some components of the nervous system scale with changes in body size [14-16]. Collectively these data suggest that some or all neuronal fates may have variable regenerative responses based on the requirement for those fates in the rescaled and smaller regenerate. However, tracking individual neuronal fates in vivo within the same individual in planarians is currently not feasible. As such, investigating dynamics that regulate neuronal subtype regeneration in this system is limited.

The anthozoan cnidarian sea anemone Nematostella vectensis is highly regenerative, capable of whole-body axis regeneration. Anecdotal observations suggest that, like planarians, Nematostella regenerates are smaller than the pre-amputated animal, and that they are capable of scaling their body size during feeding and starvation. Nematostella is an optically clear diploblastic animal whose primary axis is the oralaboral axis. The mouth is situated at the oral pole and is surrounded by tentacles that capture prey. Eight radial segments situated around the mouth run the length of the oralaboral axis [17]. The gross morphological and cellular events that occur during regeneration of the oral region have been well described. Following the initial wound healing phase, there is extensive cell death followed by an increase in proliferation and tissue rearrangements. Regeneration of the missing half of the body occurs in a stereotyped manner in 6-7 days [17-20]. How the nerve-net and individual neurons respond during regeneration have not been characterized in Nematostella. 
The Nematostella nervous system is a nerve net that is present in both the ectoderm and endoderm [21,22]. We previously identified the NvLWamide-like::mCherry transgene, which is expressed in five neuronal subtypes distributed along the oral-aboral axis in both endoderm and ectoderm, within the pharynx, and in the tentacles [23,24]. Quantification of neuronal number showed that during growth from juvenile to adults, the number of some $N v L W a m i d e-l i k e$ fates increased proportionally with length [24]. With this transgene, it is possible to quantitatively assess regeneration of neurons that are completely lost by removal of the oral region (tentacles and pharynx), and to investigate how subtypes present in the endoderm and ectoderm of the remnant fragment respond and potentially rescale. Importantly, this can be done in vivo, tracking responses within the same individual overtime. Thus, Nematostella vectensis offers the potential to identify and quantify neural subtypes throughout the process of whole-body regeneration and during size scaling.

Here we quantified responses of $N v L$ Wamide-like::mCherry+ neurons during whole-body axis regeneration and body scaling during feeding and starvation. We confirmed that Nematostella regenerates were smaller than their uncut parent, and that their nervous systems had reduced numbers of some neuronal subtypes. We observed differential regenerative responses for individual neural subtypes and classified neurons into three classes based on their specific responses. The reduced size of the regenerated nervous system led us to investigate the scalability of the nerve net. We confirmed that fed or starved Nematostella grew or shrunk respectively, and that the nervous system responded in part by modifying the number of some neuronal subtypes as the polyp changed size. Known behaviors were maintained in animals with rescaled nervous systems, which demonstrates that altering neuronal number does not reduce functionality. Collectively, these data suggest that the Nematostella nerve net is highly dynamic and scales with body size. To the best of our knowledge, these findings are the first evidence that regenerating neuronal subtypes display variable responses during whole-body axis regeneration, and are the first direct confirmation that nervous systems adjust the number of some neuron types within individuals to maintain an appropriate scale state. 


\section{Results:}

\section{Neuronal subtypes vary in regenerative response}

To investigate regenerative neurogenesis, we chose to bisect animals into two equal halves (oral and aboral remnants; see Methods) and track the response of neural subtypes in regenerating aboral remnants (Figure 1A). The choice to focus on aboral remnants is based on the fact that the regeneration timeline has been well characterized, and morphological features (e.g. tentacles and pharynx) allow for unambiguous assessment of when pre-amputated morphology is restored [17-20]. We assessed longitudinal, tripolar, pharyngeal, and tentacular neuron subtypes described by the NvLWamide-like::mCherry transgene [24]. Tentacular and pharyngeal neurons are specifically located at the oral end of the animal, whereas longitudinal and tripolar neurons are distributed along the oral-aboral axis in the endoderm and ectoderm, respectively (Figure 1B,C) [24]. $N v L$ Wamide-like::mCherry ${ }^{+}$mesentery neurons were excluded, because the ruffled mesentery structure and large number of neurons made detection and quantification unreliable without extended paralysis, which was detrimental to animal health.

We first observed regenerating aboral remnants daily to gain insight about if and when individual neural subtypes regenerated (Figure 1D; Supplemental Figure 1). Consistent with previous studies, all oral structures, including the mouth, pharynx, and tentacles, regenerated in the aboral remnant by 7 days post amputation (dpa) in 82/83 animals (Figure 1E; Supplemental Figure 1A) [18,20], and regenerates were smaller than the pre-amputated animal (Figure 1C,E; Supplemental Figure 2A,B). Pharyngeal and tentacular neurons were observed in regenerates by 72 hours post amputation (hpa) (Figure 1E; Supplemental Figure 1A,D,E). Further characterization suggested that pharyngeal neurons were first detected between 48 and $72 \mathrm{hpa}$, as those structures became recognizable (Supplementary Figure 1C,D). Longitudinal and tripolar neurons were also detected in remnant fragments, and by 48hpa longitudinal and tripolar neurons could be observed in what we believed was regenerated tissue (Supplemental Figure 1C-E). However, it was not possible to unambiguously assign longitudinal and tripolar neurons as newly regenerated or remnant neurons. 
While it is clear that new pharyngeal neurons and new tentacular neurons repopulated the newly regenerated pharynx and tentacles, we did not quantify neuronal number for these classes. Quantification of pharyngeal neurons is not possible without fixation and confocal microscopy [24]. The number of tentacular neurons is not stereotyped between tentacles in a single individual or across individuals, so we had no way to quantify the same tentacle pre- and post- amputation. For example, in one individual the number of tentacular neurons ranged from 20 to 100 neurons per tentacle (Supplemental Tables 1 and 2). We conclude that both tentacular and pharyngeal neurons regenerated, and that their regeneration coincided with the re-appearance of their corresponding tissues.

To determine if longitudinal and tripolar neurons regenerated, we quantified the number of neurons throughout regeneration (see Methods and Supplemental Figure 1F). Neurons were quantified prior to amputation (uncut), in aboral remnants immediately following amputation (time 0 cut), at 24hpa, and finally at $7 \mathrm{dpa}$ (Figure 1F-I; Supplemental Figure 3A,B). Neurons were quantified at 24hpa because extensive cell death has been reported over the first 24 hours of regeneration [25].

Initially, all regenerates were treated equally regardless of size, but regenerative responses of longitudinal neurons appeared to be size dependent. Because the number of longitudinal neurons were shown to correlate with animal length, and are evenly distributed along the oral-aboral axis (Figure 1B) [24], quantifying longitudinal neurons pre- and post-amputation was used as a proxy for length and allowed us to confirm that amputations occurred in the intended locations (see Methods). Therefore, animals were assigned to one of four different size groups based on length and the number of longitudinal neurons present in the uncut animal. Animals were categorized as small (2040 longitudinal neurons or $\sim 2.5-5 \mathrm{~mm}$ ), medium (41-60 longitudinal neurons or $\sim 3.5-5.5$ $\mathrm{mm}$ ), medium-large (61-80 longitudinal neurons or $\sim 5-7.5 \mathrm{~mm})$, and large (81-100 longitudinal neurons or $\sim 6.5-8.5 \mathrm{~mm}$ ) (Figure 1; Supplemental Figure 2A). While the measured length of each animal varied when measured once a day for one week, the number of longitudinal neurons remained constant (Supplemental Figure 2D,E). The variability in length is likely due to differences in the relaxation state of each animal when paralytic was introduced. 
Longitudinal neurons regenerated in small and medium animals, but not mediumlarge or large animals. All size categories had a decrease in longitudinal neurons between time 0 cut and 24hpa (Figure 1F,G, all $p<0.001$ ), likely due to increased cell death that occurs over the first 24hpa [25]. The number of longitudinal neurons significantly increased between 24hpa and 7dpa in small and medium animals ( $p<0.001$ for both). The numbers of longitudinal neurons in $7 \mathrm{dpa}$ regenerates were statistically similar to the numbers observed in time 0 cut animals for both small and medium groups ( small: $p=$ 0.99, medium: $p=1.00$ ), suggesting longitudinal neurons regenerated back to the numbers present in the remnant fragment at the time of bisection (Figure 1G, red and green lines). No regeneration of longitudinal neurons was observed in medium-large and large animals between 24hpa and 7dpa (Figure 1G, yellow and blue lines; $p=0.25$ and $p$ $=0.34$, respectively). Allowing regenerates to continue until 14dpa did not improve longitudinal neuron regeneration (Supplemental Figure 3C). In animals with longitudinal neuron regeneration, regenerates had less neurons than the pre-amputated (uncut) animal (Figure 1F-I; Supplemental Figure 3A,B). This is consistent with 7dpa regenerates being $\sim 65 \%$ of their pre-amputated length (Supplemental Figure $2 \mathrm{~B}$ ).

In contrast to longitudinal neurons, tripolar neurons did not regenerate in any size group. The number of tripolar neurons never returned to original uncut starting numbers (Figure 1H; Supplemental Figure 3B). The number of tripolar neurons in small animals did not change between time 0 cut, 24hpa, and 7dpa (Figure 1I, $p=1.00$ for all comparisons), indicating that small regenerates did not lose tripolar neurons by $24 \mathrm{hpa}$, nor did they gain any by $7 \mathrm{dpa}$. In medium, medium-large, and large animals, the number of tripolar neurons decreased between time 0 cut and 24hpa (Figure 1I, $p=0.001, p=$ 0.013 , and $p<0.001$, respectively). Medium and medium-large animals did not regenerate their tripolar numbers between $24 \mathrm{hpa}$ and $7 \mathrm{dpa}$ ( $p=1.00$ for both), and large animals further decreased their tripolar neurons $(p=0.055)$. Furthermore, allowing regenerates to continue to $14 \mathrm{dpa}$ did not increase tripolar neuron regeneration in animals of different sizes compared to $7 \mathrm{dpa}$ counts $(p=0.12)$. In fact, when animal size was ignored, there was a near significant decrease in the number of tripolar neurons observed between 7dpa and 14dpa ( $p=0.053$; Supplemental Figure 3D). 
We conclude that during regeneration of oral structures in bisected Nematostella, there are at least three distinct regenerative responses. The first is regeneration observed $100 \%$ of the time (e.g. pharyngeal neurons). The second is neurons do not regenerate (e.g. tripolar neurons), and the third was a variable response (e.g. longitudinal neurons).

\section{Amputation site and size of aboral remnant impacts the regeneration of longitudinal neurons}

We next sought to understand why longitudinal neurons had variable regenerative responses and considered three possibilities. One possibility is that larger animals use a different mode of regeneration from smaller animals (tissue rearrangements rather than cell proliferation). The alternative hypotheses were that the size of the remnant fragment or the size of the pre-amputated animal influenced the regenerative response of longitudinal neurons. Previous studies demonstrating Nematostella regeneration were dependent on cell proliferation and investigated younger animals with 8-10 tentacles [1820]. The larger animals in this study had 14-16 tentacles. Large group animals showed increased proliferation in both the ectoderm and endoderm of regenerating tissue (Supplemental Figure 4), suggesting that regeneration in larger animals also utilizes proliferation. However, we cannot rule out that larger regenerates may rely more heavily on remodeling existing tissues than smaller animals, which could contribute to the observed differences.

To test the hypotheses that pre-amputation size or remnant fragment size influence the regenerative response of longitudinal neurons, the amputation sites of animals of different sizes were shifted to generate larger and smaller fragments from animals in different size groups. If size of the remnant fragment influences regeneration, the shifted amputation site should alter the regenerative response. However, if preamputation size dictates regenerative potential, then altering the amputation site should not have an impact on neuronal regeneration.

Smaller aboral fragments were generated from large animals by aborally shifting the amputation site to remove $75 \%$ of the oral-aboral axis (Figure $2 \mathrm{~A}$ ). The resulting remnant fragment resembled that of a medium animal bisected in half (Figure 2D, compare black and faded green lines), and longitudinal neurons were able to regenerate 
between 24hpa and 7dpa (Figure 2C, $p=0.046$ ), which they did not do when mediumlarge or large animals bisected in half.

Orally shifting the amputation site of medium sized animals to remove only $25 \%$ of the oral-aboral axis (Figure 2B) generated remnant fragments similar to those generated by bisecting medium-large or large animals into equal halves (Figure 2E,F, compare black and faded blue and yellow lines). In this case, no longitudinal neuron regeneration was observed between $24 \mathrm{hpa}$ and $7 \mathrm{dpa}(p=1.00)$, which was similar to what was observed when medium-large and large animals were bisected in half.

When the amputation site of small animals was orally shifted (Figure 2B), the aboral remnant resembled a medium animal bisected into equal halves (Figure 2G,H, black lines). Unexpectedly, longitudinal neurons did not regenerate (Figure 2G, H, compare black and faded green lines). While the average number of longitudinal neurons did increase between $24 \mathrm{hpa}$ and $7 \mathrm{dpa}$, the change was not statistically significant ( $p=$ 0.47). It should be noted that neuronal number increased in some, but not all, regenerates. The variability in longitudinal neuron increase in small animals with orally shifted amputation sites was distinct from other experiments where the entire population more or less responded similarly. Regardless, these data suggest that the pre-amputation size of the animal was not a factor regulating longitudinal neuron regeneration. Remnant fragment size did influences longitudinal neuron regeneration, but that alone was not sufficient to explain the conditional neuronal regeneration.

Overall, these data suggest there is heterogeneity in the regenerative responses of $N v L$ Wamide-like neuronal subtypes during regeneration of oral structures that occurs in animals undergoing whole-body axis regeneration. We observed at least three different responses, which we grouped into three classes. Class I neurons regenerate $100 \%$ of the time following amputation (e.g. tentacular and pharyngeal neuron responses in this study); Class II neurons, which do not regenerate by 7dpa (e.g. tripolar neuron response in this study); and Class III neurons that have conditional regenerative responses (e.g. longitudinal neuron response in this study).

\section{Form and function are restored in regenerated Nematostella by 7 days post amputation}


Previous reports independently confirmed that normal morphology is regenerated by $7 \mathrm{dpa}$. However, the lack of regeneration of longitudinal neurons in medium-large and large animals, and the inability of tripolar neurons to regenerate in any size category, implied that the nerve net and (by extension) normal function had not yet completely regenerated by $7 \mathrm{dpa}$. To address this, we sought to determine if normal functions return by $7 \mathrm{dpa}$. If both form and function were restored by $7 \mathrm{dpa}$, it would argue that the nervous system was functional regardless of the regenerative responses of individual neuronal subtypes. At 7dpa and 14dpa, animals could catch and digest prey (Figure 3A-C). Regenerates observed at 7dpa also had 4-5 peristaltic waves on average over a 5-minute interval, with no statistical difference in the number of waves between regenerates of different size categories (Figure 3D, $p=0.35$ ). This number is similar to the number of waves observed over a 5-minute period in uncut animals ( $\mathrm{t}$-test: $\left.t_{11}=1.10, p=0.30\right)$, and in previously published observations [26]. Lastly, we tested the ability of regenerates to retract when challenged with a mechanical stimulus by developing an action-reaction poking assay using a toothpick to induce a retraction response (see Methods). Poking an animal near the mouth and tentacles induced a retraction of the animal away from the stimulus (see Supplemental Movies 1-6). We examined poking-induced behaviors in preand post-regenerated animals of all size categories. In all cases, the same animal responded to the poking assay pre-bisection (Figure 3E-H), 7dpa (Figure 3I-L), and 14dpa (Table 1). One caveat is that the function of longitudinal and tripolar neurons remains unknown, and thus we could not directly test behaviors regulated by those neurons. Nevertheless, both form and function were restored by $7 \mathrm{dpa}$, which can be considered completion of the initial regeneration response. When combined with our observations about neuronal scaling (see below and Discussion), we conclude that the lack of longitudinal regeneration in larger animals, and no regeneration of tripolar neurons in all sized animals, represents their normal regenerative response in this paradigm.

\section{Longitudinal and tripolar neuron numbers are dynamic and scale with size in adult animals}


Observations in fixed planarians suggested that some neurons alter their number to scale with size. Because regenerates were smaller than pre-amputated animals, and had a reduced number of longitudinal, tripolar neurons (Figures 1 and 2; Supplemental Figures 2 and 3), we wondered if longitudinal and tripolar numbers might fluctuate in adult animals as they grow and shrink. The number of longitudinal and tripolar neurons positively correlated with animal length [24]; Nematostella increased in size when fed [27], and they decreased in size when starved (Figure 4A,B,G). We therefore set out to determine if neuronal numbers were dynamic in adults that increased and decreased in size.

To compare responses, we performed two replicate experiments that varied the number of starting longitudinal and tripolar neurons, and thus varied the starting size of the animals (Figure 4, smaller animals; Supplemental Figure 5, larger animals). The initial experimental design was to track neuronal numbers in animals over fourteen weeks. Animals would be either starved or fed for seven weeks, then the feeding regimen would be changed for the remaining seven weeks. However, we found our largest animals that were initially fed (the feed-starve treatment group), became too large to accurately count within the time constraints of our paralytic after 3 weeks into our feeding regimen. We therefore switched the animals to starvation for the remaining 11 weeks in both independent experiments (Figure 4; Supplemental Figure 5; black lines).

After the initial 3 weeks of feeding, the fed then starved animals had significantly increased in length (Figure 4A,G, $p=0.008$ ), and the number of both longitudinal neurons (Figure 4C,D; Supplemental Figure 5A,B; $p<0.001$ for both) and tripolar neurons (Figure 4E,F, $p=0.32$; Supplemental Figure 5C,D, $p<0.001$ ) significantly increased. Following the switch to a starvation regime, the fed then starved animals significantly shrunk in size after 11 weeks (Figure 4A,G, $p=0.008$ ), and lost a significant number of longitudinal (Figure 4C,D, $p=0.003$; Supplemental Figure 5A,B, $p=0.001$ ) and tripolar neurons (Figure 4E,F, $p=0.031$; Supplemental Figure 5C,D, $p<0.001$ ).

Animals placed on the opposite feeding regimen had opposite effects on their size and neuronal numbers. Animals initially starved for 7 weeks (starved then fed group, Figure 4B) showed a trend toward a shorter length (Figure 4G, $p=0.098$ ), suggesting the animal was slower to shrink in size than grow. Starved then fed animals showed 
significant decreases in longitudinal neurons during the starvation regime (Figure $4 \mathrm{C}, \mathrm{D}, p$ $=0.004$; Supplemental Figure 5A,B; $p=0.031$ ). Tripolar neurons significantly decreased in the animals that were initially smaller to start (Figure 4E,F, $p=0.029$ ), and their decrease approached significance in animals that were larger to start (Supplemental Figure 5C,D, $p=0.094)$. When switched to feeding, the starved then fed animals significantly increased in size (Figure 4B,G, $p<0.001$ ), and both neuronal subtypes significantly increased in number (Figure 4C-F, Supplemental Figure 5A-D; $p \leq 0.001$ for all). These data suggest that Nematostella scale at least part of their nervous system by altering neuronal subtype number coincidently with increases or decreases in its size.

\section{Starved animals maintain characteristic behaviors}

Peristaltic waves were also examined to determine if starved animals maintained other normal functions. Animals starved for 6.5 weeks (Supplemental Figure 5E), and 11 weeks, still performed peristaltic movements. However, while the ability to perform peristaltic wave movements was maintained in starved animals from both timepoints, the number of waves performed during the 5-minute testing period was significantly decreased compared to the number observed pre-starvation (Supplemental Figure 5E, starved 6.5 weeks: $p=0.005$; starved 11 weeks: $p=0.014$ ). Whether this decrease in rate is due to the smaller nervous system, or lack of nutrients for a significant amount of time, has not been tested. Reproductive capability was also tested, and it was found that in both fed and starved animals, 6/6 females and 5/6 males produced and released gametes. This suggests that 6.5 weeks of starvation, and subsequent scaling down of the nerve net, does not inhibit this behavior.

To determine if the Nematostella nervous system also retained function in each scale state, the poking assay was performed in fed versus starved animals. Animals starved for 6-7 weeks responded $89.5 \%$ of the time, which was the same as when the same animals were fed normally (Figure 5; Table 1). Similarly, animals starved 11 weeks responded to the poking assay $83.3 \%$ of the time compared to a $100 \%$ response in the same animals immediately prior to the starvation period (Table 1). As expected, there was a significant decrease in the numbers of longitudinal and tripolar neurons in the starved animals (Figures 5G,H) and a significant increase in these neuronal numbers in 
the fed animals (Figure 5O,P). We conclude that retraction in response to a mechanical cue behavior is not dependent on the size of the nervous system. These preliminary findings suggest that the neurocircuitry, for at least some behaviors, are retained regardless of the overall size of the nerve net.

\section{Discussion:}

\section{Differential regeneration of neuronal subtypes during whole-body axis regeneration}

This represents the first study we are aware of to quantify responses of neuronal subtypes in vivo within individual animals undergoing whole-body axis regeneration. There was heterogeneity in the regenerative responses of $N v L$ Wamide-like::mCherry neuronal subtypes during regeneration of oral structures in whole-body axis regeneration of Nematostella. The different responses could be grouped into three classes: Class I neurons, which always regenerated; Class II neurons, which did not regenerate; and Class III neurons, whose regeneration was conditional. Essential neurons present in amputated tissues would represent Class I neurons (e.g. brain and eye neurons in planarians with anterior portions removed). Here, Class I neurons were the tentacular and pharyngeal neurons. Class II neurons have been described in populations of zebrafish tyrosine hydroxylase-positive neurons that fail to regenerate (i.e. population 12) following ablation in the zebrafish telencephalon [28]. We had difficulty identifying clear examples of Class III neurons. However, we suspect that they will be commonly found in animals whose regenerates are smaller than the precut animals, and whose nervous systems scale with body size (see below). It is important to note that each neuronal subtype's class assignment is not necessarily a property of the neuronal subtype, but rather a reflection of its behavior in a specific regeneration paradigm.

One caveat to the classification of neurons into these three classes is that the failure to observe regeneration of Class II, and sometimes Class III, neurons may reflect that regeneration was incomplete at the time of analysis. However, this is unlikely in this study for multiple reasons. First, form and function were both restored in animals by $7 \mathrm{dpa}$. Although the nervous system was reduced in overall number, all behaviors were 
present. More importantly, when nervous systems were reduced during starvation in the feed/starve experiments, their functions were not lost, suggesting that behaviors were maintained in nervous systems regardless of scale state. Second, no significant increase in neural regeneration was observed when animals were allowed to regenerate for an additional week (14dpa). By 14dpa animals had not been fed for almost three weeks. In the feed/starve experiment, the first noticeable decrease in neural number during starvation occurred in week three. Thus, we suspect that extended regeneration without eating would not improve neuronal regeneration. We do expect that feeding regenerates would increase both tripolar (Class II) and longitudinal (Class III) neurons. However, we would not characterize this as regeneration because the same phenomenon is observed in fed un-lesioned animals. The fact that $7 \mathrm{dpa}$ animals possessed the ability to feed, digest food, and display other known behaviors strongly indicates that regeneration had completed and the animals had restored functionality. We are confident that Nematostella possess at least three groups of neurons that display differential regenerative responses during regeneration of oral structures.

\section{Scalability of the Nematostella nerve net}

The correlation between neuronal number and body size suggests that Nematostella scale their nervous system with body size. A number of studies imply that nervous systems in other taxa, such as planaria, also scale by modulating cell number. In planarians, the number of visual cells and brain neurons correlate with body length, and the ratio of several types of neurons was constant regardless of overall number [14]. However, because the planarian studies were performed on fixed animals, it was not clear if neuronal number was dynamic within individual animals. We confirmed that neuronal numbers are dynamic in individual Nematostella over time. Animals gained or lost neurons as they increased and decreased in length, respectively (Figure 5). Importantly, changes in neuronal number were reversible, which argues that the nervous system senses and responds to changes in size to maintain the proper portion of neuronal cells.

The most likely mechanism for altering the number of neurons is cell proliferation and cell death. Planarians alter their total cell numbers during growth and shrinking by modulating gene expression to regulate the balance between cell death and 
proliferation [29-32]. Preliminary observations found that Nematostella starved for 3 weeks had increases in detectable anti-activated Caspase-3 and TUNNEL labeling (Eric Röttinger, IRCAN, University of Nice - Personal communication), which is consistent with increases in cell death in starved animals. Fed Nematostella show signs of increased proliferation, and in other cnidarians increases in cell proliferation are associated with growth and tissue expansion. Future studies should focus on identifying the origin of new neurons during growth, and on identifying the mechanisms that regulate the proportional increases and decreases in neuronal number that accompany changes in body size.

During both regeneration and body scaling, Nematostella retained normal body form and function. Nematostella were able to retract in response to being poked, catch and digest prey, coordinate peristaltic waves, and in the case of starved animals, perform reproductive behaviors. We can infer from these experiments that even with a smaller nerve net, these animals were capable of distinguishing between a potential predator (toothpick) and prey (brine shrimp), because while the animals responded to the poking assay with a defensive retraction, they readily captured and ingested food. Each of these behaviors likely requires multiple neuronal subtypes. However, which neurons regulate each of these behaviors is still unknown, and we do not attribute any of these behaviors to a particular neuronal subtype. Despite the limited number of functional assays in Nematostella, the range of behaviors that are unaffected by the total number of neurons suggests that the nervous system is functional in all scale states.

\section{Nervous system scale state may influence the regeneration of neuronal subtypes}

The fact that nervous systems scale with size in Nematostella, and that regenerates are smaller than pre-cut animals, suggests that the differential regenerative responses of individual neurons may be linked to the scale state of the regenerate. The two neuronal subtypes that we confirmed have reduced numbers in regenerates and scale with size are the longitudinal and tripolar neurons (Figure 4 and Supplemental Figure 5). These two subtypes represent both Class II and Class III neurons, which have two different regenerative responses (none and conditional). This suggests that the "acceptable range" of neurons needed to maintain the correct scale state varies for different neuronal subtypes. Support for this is that the variability of tripolar neurons was 
much higher than longitudinal neurons for each size group, and the distribution of tripolar neurons along the oral-aboral axis was not as uniform as longitudinal neurons, suggesting that there is more flexibility in the number and distribution of tripolar neurons required for proper function than exists for longitudinal neurons.

The conditional responses of longitudinal neuron regeneration are interesting. The data suggest that smaller remnants need to regenerate new neurons for the regenerated nervous system to be at the correct scale state, whereas larger remnants do not. Current explanations for why are speculative, but the mechanisms of regeneration may vary based on the size of the remnant fragment and amputation site. Unlike hydras, Nematostella regeneration requires cell proliferation [18]. However, evidence suggests that some remodeling of remnant tissue occurs [20]. EdU labeling suggests that larger animals still utilize cell proliferation during regeneration (Supplemental Figure 4), but perhaps they shift mechanisms to favor remodeling and smaller animals favor cell proliferation. Even if large animals do rely more heavily on remodeling, they still increase in length at equal proportions to small animals (with the caveat that length measurements are inaccurate), which we predict should result in some regeneration of longitudinal neurons. An alternative hypothesis is that the scale state relationship of longitudinal neurons to length is not linear across all sizes, with larger animals adding new neurons at a slower rate. In this case, the larger remnant fragments have a sufficient number of neurons to be within the acceptable range for that scale state. Regardless of the exact mechanism, we predict that once the key ratio of neuronal number to length for a particular neuronal subtype is identified, it should be possible to predict its regenerative response in different amputation paradigms.

The failure of longitudinal regeneration in small animals with orally shifted cut sites is difficult to explain. The remnant fragment was small enough that longitudinal regeneration was predicted by the remnant fragment size influences regenerative response model. Intriguing to us was the fact that the response of small animals with orally shifted cut sites was much more heterogenous than the other regeneration experiments. The orally shifted cut sites in small animals was proximal to the mouth and pharynx, whereas other amputations performed in this study were much more distal to the oral structures. It is possible that oral amputations close enough to the mouth resemble loss of a particular 
structure (i.e. amputation of limb) rather than whole-body axis regeneration, and subsequently the regenerative mechanisms are distinct. This could explain the variability in our data and may underscore the fact that neuronal subtypes may be classified differently depending on the amputation paradigm. Because this is the first detailed investigation of regenerative neurogenesis in Nematostella, it is clear that additional studies are needed to better understand the mechanisms regulating neuronal responses.

\section{Conclusion:}

The ability to observe and quantify neuronal subtypes make Nematostella a powerful model to investigate neurogenesis in the context of both regeneration and whole-body scaling. Our data suggest that the regenerative responses of neuronal subtypes in Nematostella are variable during regeneration of oral structures, and that the nervous system scales with size. We suspect that variable neuronal responses are linked to regenerates rescaling their nervous system to accommodate their smaller size, but additional studies are necessary to validate this hypothesis. How Nematostella control the regeneration and incorporation of new neurons into the existing nerve net, and how they scale the numbers of these neurons to body size in real time, remains unknown, but both are exciting areas for future investigation. Lastly, our data imply that experiments aimed at uncovering the molecular mechanisms regulating neural regeneration in Nematostella require careful consideration of the differential regenerative prioritization of neuronal subtypes associated with each amputation paradigm when designing experiments and interpreting results.

\section{Methods:}

\section{Animal care}

All animals were kept at room temperature and fed rotifers or crushed brine shrimp until they reached the young juvenile adult stage, at which time they were transferred to $17^{\circ} \mathrm{C}$ and switched to solely brine shrimp. All animals were housed at $17^{\circ} \mathrm{C}$ in the dark and fed brine shrimp $4 \mathrm{x} /$ week, unless otherwise noted for experimental 
purposes. Nematostella were maintained in 1/3x artificial sea water, (Nematostella medium) at a $\mathrm{pH}$ of 8.1-8.2 that was changed once per week.

Nematostella were relaxed in $7.14 \%$ (wt/vol) $\mathrm{MgCl}_{2}$ in Nematostella medium for $\sim 10$ minutes prior to neural quantification, imaging, or bisection. No paralytic was used during behavioral experiments. The lengths of relaxed animals were measured in $\mathrm{mm}$ with a ruler placed under a Petri dish containing the animal.

\section{Quantification and imaging of NvLWamide-like::mCherry-expressing animals}

To quantify longitudinal and tripolar neuron numbers, we first determined that the number of neurons in each radial segment were similar to one another within each individual animal (Supplemental Tables 3 and 4). The minimal variation allowed us to quantify neuronal number in an individual by calculating the representative number of longitudinal and tripolar neurons present by averaging 2-4 segments for each animal/time point (Supplemental Figure 1F). The same individual was kept isolated so that the number of neurons present within one animal could be tracked over time.

NvLWamide-like::mCherry tripolar and longitudinal neurons were counted on live animals under a dissection microscope (Nikon SMZ1270), on live animals placed under a slide with a raised coverslip on a compound microscope (Nikon NTi), or on full-length images of Nematostella captured on a Nikon Nti. All quantifications were done by hand, because it was more reliable than images acquired on a fluorescent compound scope. These animals were too large to generate confocal montages covering the whole animal in 20 minutes or less, which is the upper limit of the amount of time these animals tolerate in the paralytic with no detrimental effects.

\section{Imaging}

Live images of $N v L$ Wamide-like::mCherry-expressing animals were taken on a Nikon NTi with a Nikon DS-Ri2 color camera with Nikon Elements software. Large fulllength images are composed of several images stitched together. Confocal images of EdU staining was captured using a Zeiss LSM 880 with LSM software and processed using Imaris 8.4.1 software (Bitplane LLC) to create 3D images from serial optical sections (zstacks). Images were cropped and assembled using Photoshop and Illustrator (Adobe). 
Live video was captured on a Nikon Eclipse E1000 microscope with Nikon Imaging software or a Nikon dissection microscope (Nikon SMZ1270) with a USB camera attachment. Movies were processed with ImageJ/Fiji software and screen shots were obtained from individual frames, then cropped and assembled using Adobe Photoshop and Illustrator.

\section{Regeneration experiments}

For the quantification of longitudinal and tripolar NvLWamide-like::mCherry expressing neuronal subtypes during regeneration, only animals containing 20-100 longitudinal neurons were used, which were typically 2.5 to $9 \mathrm{~mm}$ long (Supplemental Figure 2A). This size range represents the sizes of naturally occurring wild-caught animals found along the East Coast of the United States (Adam Reitzel, University of North Carolina, Charlottesville, NC, personal communication, 11/2018).

Longitudinal and tripolar neurons were quantified first on the uncut animal, then immediately following bisection (time 0 cut), 24 hours post amputation (hpa), and 7 days post amputation (dpa). For some regenerating animals, additional counts were conducted at 14dpa. Animals were either bisected in half with the cut site at the oral-aboral midpoint ( $\sim 50 \%$ body removed), bisected below the pharynx ("oral shift", $\sim 25 \%$ removed), or bisected at the aboral end ("aboral shift", 75\% removed) (see above).

We developed a strategy to ensure the amputation sites were reproducibly positioned in the middle $50 \%$ of the animal, oral $75 \%$ of the animal, or aboral $25 \%$ of the animal. Longitudinal neurons are equally distributed along the oral-aboral axis (Figure 1B). Thus, confirmation of cut sites were achieved by comparing the number of neurons in the remnant fragment immediately after amputation with the number of total neurons present in the precut animal.

Following quantification and/or bisection, the remnant aboral fragment was washed with Nematostella medium, then placed in the dark at room temperature. Prior to quantification, animals were starved for 3-4 days [18,20]. Animals were not fed for the duration of the regeneration experiments.

\section{Feed/Starve experiments}


For each animal, the number of longitudinal and tripolar neurons were counted at time 0, at the time of their feeding regime switch (Starved then fed: 6-7 weeks later; Fed then starved: 3 weeks later), and at the end of the 14 week experiment (Starved then fed: 6-7 weeks later; Fed then starved: 11 weeks later). All animals were maintained on the same normal feeding schedule until time 0 of the experiment. At this point, animals were randomly assigned to either the starve-feed group or feed-starve group and their initial starting neural counts were taken. Animals on the feeding regime were fed brine shrimp 2x/week plus oyster 1x/week, and were given weekly water changes.

\section{Poking assay}

Animals were transferred to individual dishes containing Nematostella medium and observed under a dissection microscope. Once the animal had relaxed its tentacles, a toothpick was slowly inserted into the medium so as to not disturb the animal prematurely. The animal was then "poked" with the tip of the toothpick at the oral region. The entire assay was video recorded and analyzed to determine the animal's response. Following the "poking assay", the animal was left alone in the dish until it relaxed again, upon which time the poking assay was performed again. The assay was performed 3 consecutive times. A positive response was determined if the animal responded to being touched with a toothpick by retracting some or all of its tentacles, scrunching or retracting in its mouth (oral region), or retracting its entire body. To be considered responsive, the animal had to react in at least 1 out of the 3 poking assays performed. The poking assay was done in a repeated fashion, with each animal being tested at the beginning and end of the experiment.

\section{Peristaltic Waves}

Animals were individually placed in dishes filled with Nematostella medium and observed under a dissection scope. Data collection did not begin until animals relaxed their tentacles and/or at least $75 \%$ of their body-column. Once relaxed, animals were video recorded for 5 continuous minutes. Recordings were then analyzed for the number of peristaltic waves that occurred during this time. A peristaltic wave was defined as an inward radial contraction that propagated down the body column in an oral to aboral 
direction [26]. Waves that were in progress at the start of data collection were counted, and so were waves that did not go to completion by the end of the observation period. In the regeneration experiment, the number of waves observed/5 minutes was compared between animals categorized as small, medium, medium-large, and large animals. In the feed/starve experiment, animals were observed at the beginning and end of the starvation period for whether any peristaltic wave behavior occurred or not.

\section{Feeding behavior}

Animals were transferred to individual dishes filled with Nematostella medium and observed using a dissection microscope. Once an animal relaxed its tentacles, freshly hatched brine shrimp (5-10) were added to the dish. Animals were first observed for their ability to capture and ingest brine shrimp. Once at least one was captured, the rest of the brine shrimp were removed and the animal was left overnight. The following day, the dish was checked for evidence of expelled brine shrimp that had not been digested following capture and ingestion.

\section{Reproductive behavior}

Animals were kept individually in dishes filled with Nematostella medium. Each individual was spawned prior to the starvation period to determine their gametic sex. Those that released eggs were designated as female and those that released sperm were designated as male. Egg clutches are visible to the naked eye and are therefore easily scored for their presence or absence. Sperm release was determined by observing if a clutch of eggs from an isolated known female would be fertilized (scored by the presence of cleaving embryos) when given water from an unidentified individual's dish. As a false-positive control, half of each known female's eggs were set aside and observed to make sure no development occurred. Experimental animals were starved for 6 weeks while control animals were fed artemia once per week over six weeks. All animals were kept in the dark at $17^{\circ} \mathrm{C}$ and received weekly water changes. After six weeks, all animals were placed on a light box in order to stimulate reproductive behavior. Whether or not animals released gametes was observed using the methods described above. 


\section{EdU Stain}

EdU incorporation was carried out as previously described [18]. Animals were incubated with $330 \mathrm{uM}$ EdU in Nematostella medium for 30 minutes, then fixed as previously described and dehydrated to $100 \% \mathrm{MeOH}$ and stored at $-20{ }^{\circ} \mathrm{C}$. Following rehydration, samples were permeabilized in PBT (PBS with 0.5\% Triton X-100, Sigma) for $\sim 2$ hours. Animals were then treated with the reaction cocktail (prepared following the Click-It protocol provided by the manufacturer, with the exception of doubling the amount of Alexa Fluor azide) at room temperature in the dark for 20 minutes. Following 5 long washes in PBT, the animals were labeled with propidium iodide for 1 hour while rocking, washed 5X in PBT, and finally transferred to 90\% glycerol for imaging.

\section{Statistical analysis}

For the regeneration and feed/starve experiments, mixed analysis of variance (ANOVA) tests were used to analyze each dependent variable (i.e. the number of longitudinal and tripolar neurons quantified). For both experiments, each observation of an individual over time served as the within-subject factor (Regeneration experiment: time 0 cut, 24hpa, 7dpa; Feed/starve experiment: week 1, week of feeding regime switch, week 14). The between subject factors used were animal size category (small: 20-40mm, medium: 41-60mm, medium-large: 61-80mm, large: $81-100 \mathrm{~mm}$ ) and assigned feeding group (Feed-Starve or Starve-Feed) for the regeneration and feed/starve experiments, respectively. A mixed ANOVA was also used to analyze changes in oral-aboral length during the feed/starve experiment, with each observation of an individual serving as the within-subject factor (week 1, week of feeding regime switch, and week 14) and assigned feeding group as the between-subject factor (Feed-Starve or Starve-Feed). Data used for ANOVA tests met assumptions of normality and homogeneity. Greenhouse-Geisser corrected F-statistics were reported if sphericity was violated (i.e. Figure 1 G,I). When omnibus ANOVA results were significant, post-hoc testing was performed using Bonferroni corrections in order to maintain tight control over the type 1 error rate. Effect sizes are reported as partial eta ${ }^{2}\left(\eta_{\mathrm{p}}{ }^{2}\right)$. Full ANOVA model results are reported in Supplemental Document 1 for all significant omnibus analyses. Paired t-tests were used 
to compare individual changes in the number of neurons or length at two time points. All data were analyzed using SPSS version 25.0.

\section{Acknowledgements}

We would like to thank Aldine Amiel and Eric Röttinger for critically reading this manuscript and providing feedback, as well as their personal communications regarding cell death in starved animals. We also thank Adam Reitzel for providing insights about what size ranges we should target to represent adult animals found in the wild. We thank David Balli and Omar Ahmed for providing help with R software and construction of the line graphs. This work was funded by the National Institutes of Health Award Number R01GM127615.

\section{Competing Interests Statement}

The authors declare that they have no financial or non-financial competing interests.

Table 1: Summary of the analysis for the action-reaction poking assay in regenerated, starved, and fed animals.

\begin{tabular}{|c|c|c|c|c|}
\hline $\begin{array}{c}\text { Experimental } \\
\text { treatment }\end{array}$ & \multicolumn{2}{|c|}{ Timepoint } & $\begin{array}{l}\text { Number to respond } \\
\text { to touch stimulus }\end{array}$ & $\begin{array}{l}\text { Percent to respond to } \\
\text { touch stimulus }\end{array}$ \\
\hline \multirow{12}{*}{ Regeneration } & \multirow{4}{*}{ Uncut } & Small & $5 / 5$ & $100 \%$ \\
\hline & & Medium & $6 / 6$ & $100 \%$ \\
\hline & & Medium-large & $5 / 5$ & $100 \%$ \\
\hline & & Large & $6 / 6$ & $100 \%$ \\
\hline & \multirow{4}{*}{$7 \mathrm{dpa}$} & Small & $5 / 5$ & $100 \%$ \\
\hline & & Medium & $6 / 6$ & $100 \%$ \\
\hline & & Medium-large & $5 / 5$ & $100 \%$ \\
\hline & & Large & $6 / 6$ & $100 \%$ \\
\hline & \multirow{4}{*}{14 dpa } & Small & $5 / 5$ & $100 \%$ \\
\hline & & Medium & $6 / 6$ & $100 \%$ \\
\hline & & Medium-large & $5 / 5$ & $100 \%$ \\
\hline & & Large & $6 / 6$ & $100 \%$ \\
\hline \multirow{2}{*}{ Starvation } & \multicolumn{2}{|l|}{ Time 0} & $17 / 19$ & $89 \%$ \\
\hline & \multicolumn{2}{|c|}{ Starved 6-7 weeks } & $17 / 19$ & $89 \%$ \\
\hline \multirow{2}{*}{ Feeding } & \multicolumn{2}{|l|}{ Time 0} & $12 / 12$ & $100 \%$ \\
\hline & \multicolumn{2}{|c|}{ Fed 3 weeks } & $10 / 12$ & $83 \%$ \\
\hline
\end{tabular}




\section{Figure Legends:}

\section{Figure 1: Differential regeneration of $N v L$ Wamide-like neurons depends on the} specific neuronal subtype. (A) Schematic for oral bisection at $\sim 50 \%$ body length. (B) Quantification of the number of longitudinal neurons along the oral-aboral axis in each of the 4 equal quarters. Each line represents an individual animal $(n=10)$. (C) Uncut NvLWamide-like::mCherry expressing animal. (D) Same animal as $\mathrm{C}$ at time 0 cut. (E) Same animal as $\mathrm{C}$ and $\mathrm{D}$ at 7 days post amputation (dpa), with regenerated oral structures. Arrows indicate longitudinal neurons and arrowheads indicate tripolar neurons. (F-I) Quantification of longitudinal and tripolar neurons before amputation (uncut), immediately following bisection at the O-A midline (time 0 cut), 24hpa, and at completion of regeneration $(7 \mathrm{dpa})$. Lines represent the average number of neurons per radial segment in small (red), medium (green), medium-large (orange), and large (blue) sized animals. Data points represent means \pm SEM. The numbers of longitudinal neurons (F) or tripolar neurons $(\mathrm{H})$ never regenerated back to their original pre-cut numbers. $(\mathrm{G})$ Longitudinal neurons regenerated back to numbers similar to time 0 cut in the small and medium sized animals, but did not regenerate in the larger animals by $7 \mathrm{dpa}(\mathrm{n}=29$ small, 20 medium, 16 medium-large, 18 large). (I) Tripolar neurons did not regenerate by $7 \mathrm{dpa}$ ( $\mathrm{n}=28$ small, 19 medium, 16 medium-large, 18 large). Data were analyzed using repeated measure ANOVAs: $(\mathrm{G})$ longitudinal neurons, $F_{5.1,134.4}=13.46, p<0.001, \eta_{\mathrm{p}}{ }^{2}=$ 0.34; (I) tripolar neurons: $F_{4.5,116.2}=3.93, p=0.003, \eta_{\mathrm{p}}{ }^{2}=0.13$. Asterisks identify significant regeneration between $24 \mathrm{hpa}$ and $7 \mathrm{dpa}$. Scale bar $=1 \mathrm{~mm}$.

\section{Figure 2: Regeneration of longitudinal neurons depends on the size of the} regenerating fragment. (A-B) Schematics demonstrating the shifts in cut sites used to obtain remnant fragments of varying sizes. (C-H) Quantification of longitudinal neurons in regenerates with shifted cut sites at time 0 cut, $24 \mathrm{hpa}$, and $7 \mathrm{dpa}$. Regenerates from an aborally-shifted cut site in large animals regenerated their longitudinal neurons (C), resembling regenerates of small and medium sized animals cut at the midline of the O-A axis (D). Regenerates from an orally-shifted cut site in medium animals did not regenerate their longitudinal neurons $(\mathrm{E})$, resembling regenerates from large animals cut 
at the midline of the O-A axis (F). Regenerates from an orally-shifted cut site in small animals did not regenerate their longitudinal neurons $(\mathrm{G})$, which is different from medium animals cut at the midline of the O-A axis $(\mathrm{H})$. Data were analyzed using repeated measure ANOVAs comparing observation times: (C) Large with aborally shifted cut site, $F_{2,14}=5.58, p=0.017, \eta_{\mathrm{p}}{ }^{2}=0.44, \mathrm{n}=8$; (E) Medium with orally shifted cut site, $F_{2,24}=21.67, p<0.001, \eta_{\mathrm{p}}^{2}=0.64, \mathrm{n}=13$; (G) Small with orally shifted cut site, $F_{2,24}=8.56, p=0.002, \eta_{\mathrm{p}}{ }^{2}=0.42, \mathrm{n}=13$ (see Supplemental Document 1 for full model results). Data points in $\mathrm{C}-\mathrm{H}$ represent means $\pm \mathrm{SEM}$.

Figure 3: Normal behaviors are restored in regenerated animals by 7dpa. Representative regenerated animal pre-feeding (A), during feeding (B), and 3 hours post feeding (C) at 7dpa. Asterisks identify the locations of brine shrimp during capture, ingestion, and digestion. Note the increase in orange pigment in the mesenteries due to the digestion of the brine shrimp. All regenerates were capable of capturing, ingesting, and digesting prey ( $\mathrm{n}=6 / 6$ small; $5 / 5$ medium; $5 / 5$ medium-large; $6 / 6$ large). Arrows in A and $\mathrm{C}$ highlight peristaltic wave movements. (D) Animals in all size categories carried out similar numbers of peristaltic waves by $7 \mathrm{dpa}$ (one-way ANOVA: $F_{3,17}=1.16, p=$ $\left.0.35, \eta_{\mathrm{p}}{ }^{2}=0.17\right)$. (E-L) Response to the poking assay is maintained in regenerated animals ( $\mathrm{n}=5 / 5$ small, 6/6 medium, 5/5 medium-large, 6/6 large). (E-H) Nematostella responded to the touch stimulus by retracting its tentacles when touched with a toothpick. (I-L) At 7dpa, the same animal again responded to being poked with a toothpick by retracting its tentacles.

\section{Figure 4: The Nematostella nerve net scales with changes in size.}

(A-B) Visualization of $N v L$ Wamide-like neurons in relation to body size during periods of feeding and starvation. (C-F) Quantification of $N v L$ Wamide-like neurons during periods of growth and starvation with both mean $(C, E)$ and population $(D, F)$ data presented $(n=$ 10). Animals fed for 3 weeks rapidly grew longer in length (A, 3 weeks fed), and numbers of both longitudinal $(\mathrm{C}, \mathrm{D}$, black lines) and tripolar $(\mathrm{E}, \mathrm{F}$, black lines) neurons significantly increased. Following 11 weeks of starvation, the same animal then shrunk in 
size (A, 11 weeks starved) and the numbers of both longitudinal and tripolar neurons significantly decreased (C-F, black lines). Animals initially starved for 7 weeks shrunk in size (B, 7 weeks starved) and the numbers of both longitudinal (C, D, blue lines) and tripolar (E, F, blue lines) neurons significantly decreased. Upon being switched to the feeding regime for 7 weeks, the same animals grew longer (B, 7 weeks starved) and the numbers of both longitudinal and tripolar neurons significantly increased (C-F, blue lines). Oral end is to the left. Scale bar $=1 \mathrm{~mm} . \mathrm{n}=5$ animals per treatment group. Arrows indicate longitudinal neurons and arrowheads indicate tripolar neurons. Longitudinal and tripolar neuron data were analyzed using mixed ANOVAs to look for interactions between assigned feeding regime and observation times: (C) Longitudinal, $F_{2,16}=102.01, p<0.001, \eta_{p}{ }^{2}=0.93$; (E) Tripolar, $F_{2,16}=48.60, p<0.001, \eta_{p}^{2}=0.86$. (G) The measured length of the animals significantly decreased during the starvation period and increased during the feeding period. Length data were analyzed with a mixed ANOVA to look for an interaction effect between assigned feeding regime and observation time: $F_{2,34}=25.75, p<0.001, \eta_{\mathrm{p}}{ }^{2}=0.60$ (see Supplemental Document 1 for full model results). Data points in $\mathrm{C}, \mathrm{E}$, and $\mathrm{G}$ represent means $\pm \mathrm{SEM}$.

\section{Figure 5: Starved and fed animals respond to the poking assay by retracting their}

tentacles and body. (A-E) A normally fed animal responded to the touch stimulus from a toothpick by retracting its tentacles and oral region $(n=17 / 19)$. (D-E) Following 6-7 weeks starvation, the same animal again responded to being touched with a toothpick by retracting its tentacles and oral region $(\mathrm{n}=17 / 19)$. $(\mathrm{F}-\mathrm{G})$ Quantification of the number of longitudinal and tripolar neurons in individual animals following each poking experiment. The numbers of both longitudinal (I: $t_{16}=7.97, p<0.001, \eta_{\mathrm{p}}{ }^{2}=0.80$ ) and tripolar $\left(\mathrm{J}: t_{16}=6.99, p<0.001, \eta_{\mathrm{p}}{ }^{2}=0.75\right)$ neurons significantly decreased after $6-7$ weeks starvation. (H-J) A normally fed animal responded to the touch stimulus from a toothpick by retracting its tentacles and body $(n=13 / 13)$. $(K-M)$ Following 3 weeks feeding, the same animal again responded to being touched with a toothpick by retracting its tentacles and oral region $(n=12 / 13)$. $(\mathrm{N}-\mathrm{O})$ Quantification of the number of longitudinal and tripolar neurons in individual animals following each poking 
experiment. The numbers of both longitudinal (G: t-test: $t_{12}=-9.15, p<0.001, \eta_{\mathrm{p}}{ }^{2}=0.88$ ) and tripolar (H: t-test: $\left.t_{12}=-12.76, p<0.001, \eta_{\mathrm{p}}{ }^{2}=0.93\right)$ neurons significantly increased following 3 weeks feeding.

\section{Supplemental Figure 1. Regeneration of oral structures and neurons in NvLWamide-like::mCherry animals following bisection along the O-A axis.}

(A) Regenerated oral structures, including a mouth, pharynx (arrow), and tentacles (arrowhead) at 7dpa. (A') New neurons are observed in the regenerated oral structures, including pharyngeal (arrow) and tentacular (arrowhead) neurons. (B) Remnant fragment at 24hpa. No new structures were observed at this time point. (C) Tentacle buds were visible in the remnant fragment at 48hpa. Longitudinal (green arrowhead) and tripolar (purple arrowhead) neurons were observed. Regenerated pharyngeal neurons were also present in the regenerating pharynx (orange arrowhead) at 48hpa. (D) Pharyngeal (orange arrowhead) and tentacular (red arrowhead) neurons were clearly visible in these regenerating structures by $72 \mathrm{hpa}$. (E) Clearly regenerated pharyngeal (orange arrowhead) and tentacular (red arrowhead) neurons are seen at 96hpa. Longitudinal neurons (green arrowhead) and tripolar neurons (purple arrowhead) also populated the regenerated tissue, but whether these were regenerated neurons or from the remnant was undetermined. Asterisks indicate oral opening. (F) Example of how longitudinal (green arrowheads) and tripolar (purple arrowheads) were quantified within a longitudinal track and radial segment, respectively. This was repeated for 2-4 tracks or radial segments and averaged to determine the number/animal. Note that for regeneration and feed-starve experiments, neuron counts were performed live under a dissection scope and not from images.

\section{Supplemental Figure 2: Length measurements and neural quantifications in}

Nematostella. (A-C) Regenerated animals never re-grew back to their original length, and there was no difference in the percent length of the regenerates relative to their body size by 7dpa. (A) Population data showing lengths for individual animals before they were cut (uncut) and 7dpa. Small animals are represented in red, medium in green, medium-large in orange, and large in blue $\left(t_{30}=8.65, p<0.001, \eta_{\mathrm{p}}{ }^{2}=0.71, \mathrm{n}=31\right)$. (B) 
Percent length regained in 7dpa animals bisected in half did not differ between animals of different size categories (one-way ANOVA: $F_{3,27}=0.23, p=0.875, \eta_{\mathrm{p}}{ }^{2}=0.025, \mathrm{n}=11$ small; 8 medium; 6 medium-large; 6 large). (C) Percent length regained in 7dpa animals with shifted cut sites varied (one-way ANOVA: $F_{2,28}=10.92, p<0.001, \eta_{\mathrm{p}}{ }^{2}=0.44, \mathrm{n}=$ 13 small oral shift; 11 medium oral shift; 7 large aboral shift; see Supplemental Document 1). Bars in B and C represent means \pm SEM. (D) Length measurements taken over 5 consecutive days show high variability. (D) The number of longitudinal neurons did not change over the same 5 day period as (D). In D and E, each line represents an individual animal.

\section{Supplemental Figure 3: Quantification of neuronal numbers during oral}

regeneration. Population data showing the time course for the average number of (A)

longitudinal neurons and (B) tripolar neurons per 2-4 radial segments in individual regenerating animals bisected in half. Neuronal numbers did not return to levels present in the uncut animal. Quantifications were taken before cutting (uncut), time 0 cut, 24hpa, and 7dpa ( $\mathrm{n}=83$ animals). Data represented in A and B are the same dataset grouped by starting size and averaged in Figure 1. (C) Longitudinal neurons did not increase in number between 7 and 14dpa in animals of any size category (mixed ANOVA: observation time x size: $F_{3,42}=0.86, p=0.47, \eta_{\mathrm{p}}^{2}=0.06$ ). (D) Tripolar neurons did not increase in number between $7 \mathrm{dpa}$ and $14 \mathrm{dpa}$ when comparing animals of different sizes (mixed ANOVA: observation time x size, $F_{3,42}=2.04, p=0.122, \eta_{\mathrm{p}}{ }^{2}=0.13$ ). Regardless of size, there was a near significant decrease in tripolar neurons between $7 \mathrm{dpa}$ and 14dpa (mixed ANOVA: observation time, $F_{1,42}=3.97, p=0.053, \eta_{\mathrm{p}}^{2}=0.09$. (C) $\mathrm{n}=$ 12 small; 12 medium; 10 medium-large; 12 large. (D) $\mathrm{n}=12$ small; 12 medium; 9 medium-large; 11 large.

\section{Supplemental Figure 4: Detection of proliferating cells in "large" Nematostella}

during oral regeneration. (A) EdU (green) is detected at 48hpa in the oral-most region of large regenerating animals bisected in half. (B) EdU overlaid with propidium iodide (PI, red) stain in the same animal as A, with yellow color showing co-expression. (C) 
Higher magnification image of the same animal, showing EdU detection in both the ectoderm and endoderm, suggesting both tissue layers proliferate during oral regeneration ( $n=3$ large animals). Scale bars for A and B =200um; $C=50 u m$.

\section{Supplemental Figure 5: Independent experiment demonstrating Nematostella nerve} net scales with changes in size. (A-D) Quantification of $N v L$ Wamide-like neurons during periods of feeding and starvation with both mean $(\mathrm{A}, \mathrm{C})$ and population $(\mathrm{B}, \mathrm{D})$ data presented ( $\mathrm{n}=10,5$ animals per treatment group). The numbers of both longitudinal (A, $\mathrm{B}$, black lines) and tripolar ( $\mathrm{C}$ and $\mathrm{D}$, black lines) neurons significantly increased with 3 weeks of feeding, and then both longitudinal and tripolar neurons significantly decreased with 11 weeks starvation (A-D, black lines). The numbers of both longitudinal (A, B, blue lines) and tripolar (C, D, blue lines) neurons significantly decreased following 6 weeks starvation. Upon being switched to the feeding regime for 8 weeks, the numbers of both longitudinal and tripolar neurons significantly increased (A-D, blue lines).

Longitudinal and tripolar neuron data were analyzed using mixed ANOVAs to look for interactions between assigned feeding regime and observation times: (A) Longitudinal, $F$ $2,16=38.88, p<0.001, \eta_{\mathrm{p}}{ }^{2}=0.83$; (C) Tripolar, $F_{2,16}=206.13, p<0.001, \eta_{\mathrm{p}}{ }^{2}=0.96$ (see Supplemental Document 1 for full model results). (E) Starved animals maintained peristaltic movements along the body column. Data points in A, C, and E represent mean \pm SEM. 


\section{References}

1. Cebrià F, Nakazawa M, Mineta K, Ikeo K, Gojobori T, Agata K. Dissecting planarian central nervous system regeneration by the expression of neural-specific genes.

Development, Growth \& Differentiation. 2002;44:135-46.

2. Currie KW, Pearson BJ. Transcription factors $1 \mathrm{hx} 1 / 5-1$ and pitx are required for the maintenance and regeneration of serotonergic neurons in planarians. Development. $2013 ; 140: 3577-88$.

3. Chen CCG, Wang IE, Reddien PW. pbx is required for pole and eye regeneration in planarians. 2013;140:719-29.

4. Deochand ME, Birkholz TR, Beane WS. Temporal regulation of planarian eye regeneration. Regeneration. 2016;3:209-21.

5. Gahan JM, Schnitzler CE, DuBuc TQ, Doonan LB, Kanska J, Gornik SG, et al. Functional studies on the role of Notch signaling in Hydractinia development. Dev. Biol. Elsevier Inc; 2017;428:224-31.

6. Galliot B, Miljkovic-Licina M, Ghila L, Chera S. RNAi gene silencing affects cell and developmental plasticity in hydra. Comptes Rendus Biologies. 2007;330:491-7.

7. Miljkovic-Licina M, Chera S, Ghila L, Galliot B. Head regeneration in wild-type hydra requires de novo neurogenesis. 2007;134:1191-201.

8. Siebert S, Farrell JA, Cazet JF, Abeykoon Y, Primack AS, Schnitzler CE, et al. Stem cell differentiation trajectories in Hydra resolved at single-cell resolution. 2018;:1-29.

9. Roberts-Galbraith RH, Brubacher JL, Newmark PA. A functional genomics screen in planarians reveals regulators of whole-brain regeneration. eLIFE. 2016;:1-31.

10. Randolph H. Observations and Experiments on Regeneration in Planarians. 1897. pp. $1-31$.

11. Reddien PW. The Cellular and Molecular Basis for Planarian Regeneration. Cell. Elsevier Inc; 2018;175:327-45.

12. Thommen A, Werner S, Frank O, Phillipp J, Knittelfelder O, Quek Y, et al. Body size-dependent energy storage causes Kleiber's law scaling of the metabolic rate in planarians. eLIFE. 2019;:1-29.

13. Birkholz TR, Van Huizen AV, Beane WS. Staying in shape: Planarians as a model for understanding regenerative morphology. Seminars in Cell \& Developmental Biology. Elsevier Ltd; 2019;87:105-15. 
14. Takeda H, Nishimura K, Agata K. Planarians Maintain a Constant Ratio of Different Cell Types During Changes in Body Size by Using the Stem Cell System. Zoological Science. 2009;26:805-13.

15. Oviedo NJ, Newmark PA, Sánchez Alvarado A. Allometric scaling and proportion regulation in the freshwater planarian Schmidtea mediterranea. Dev. Dyn. 2003;226:32633.

16. Hill EM, Petersen CP. Wnt/Notum spatial feedback inhibition controls neoblast differentiation to regulate reversible growth of the planarian brain. Development. 2015;142:4217-29.

17. Layden MJ, Rentzsch F, Röttinger E. The rise of the starlet sea anemone Nematostella vectensis as a model system to investigate development and regeneration. WIREs Dev Biol. John Wiley \& Sons, Inc; 2016;:1-21.

18. Passamaneck YJ, Martindale MQ. Cell proliferation is necessary for the regeneration of oral structures in the anthozoan cnidarian Nematostella vectensis. BMC Dev Biol. BioMed Central; 2012;12:34.

19. Bossert PE, Dunn MP, Thomsen GH. A staging system for the regeneration of a polyp from the aboral physa of the anthozoan Cnidarian Nematostella vectensis. Dev. Dyn. 2013;242:1320-31.

20. Amiel AR, Johnston HT, Nedoncelle K, Warner JF, Ferreira S, Röttinger E.

Characterization of Morphological and Cellular Events Underlying Oral Regeneration in the Sea Anemone, Nematostella vectensis. IJMS. 2015;16:28449-71.

21. Rentzsch F, Layden M, Manuel M. The cellular and molecular basis of cnidarian neurogenesis. WIREs Dev Biol. 2016;:1-19.

22. Nakanishi N, Renfer E, Technau U, Rentzsch F. Nervous systems of the sea anemone Nematostella vectensis are generated by ectoderm and endoderm and shaped by distinct mechanisms. Development. Oxford University Press for The Company of Biologists Limited; 2012;139:347-57.

23. Layden MJ, Johnston H, Amiel AR, Havrilak J, Steinworth B, Chock T, et al. MAPK signaling is necessary for neurogenesis in Nematostella vectensis. BMC Biol. BioMed Central; 2016;14:61.

24. Havrilak JA, Faltine-Gonzalez D, Wen Y, Fodera D, Simpson AC, Magie CR, et al. Characterization of NvLWamide-like neurons reveals stereotypy in Nematostella nerve net development. Dev. Biol. Elsevier Inc; 2017;:1-0.

25. Warner JF, Amiel AR, Johnston H, Röttinger E. Regeneration is a partial redeployment of the embryonic gene network. bioRxiv. Cold Spring Harbor Laboratory; 2019;121:1731-28. 
26. Faltine-Gonzalez DZ, Layden MJ. Characterization of nAChRs in Nematostella vectensis supports neuronal and non-neuronal roles in the cnidarian-bilaterian common ancestor. Evodevo. BioMed Central; 2019;:1-18.

27. Hand C, Uhlinger KR. The Culture, Sexual and Asexual Reproduction, and Growth of the Sea Anemone Nematostella vectensis. Biological Bulletin. 1992;182:169-76.

28. Caldwell LJ, Davies NO, Cavone L, Mysiak KS, Semenova SA, Panula P, et al. Regeneration of Dopaminergic Neurons in Adult Zebrafish Depends on Immune System Activation and Differs for Distinct Populations. J. Neurosci. 2019;39:4694-713.

29. Baguñà J, Romero R, Salo E, Collet J, Auladell C, Ribas M, et al. Growth, Degrowth, and Regeneration as Developmental Phenomena in Adult Freshwater Planarians. In: Marthy HJ, editor. Experimental Embryology in Aquatic Plants and Animals. 1990. pp. 129-62.

30. Baguñà J, Romero R,. Quantitative analysis of cell types during growth, degrowth, and regeneration in the planarians Dugesia mediterranea and Dugesia tigrina. Hydrobiologia. 1981;84:181-94.

31. Romero R, Baguñà J. Quantitative cellular analysis of growth and reproduction in freshwater planarians (Turbellaria; Tricladida). I. A cellular description of the intact organism. Invertebrate Reproduction \& Development. 1991;19:157-65.

32. Pascual-Carreras E, Marin-Barba M, Herrera-Úbeda C, Font-Martín D, Eckelt K, de Sousa N, et al. Planarian cell number depends on blitzschnell, a novel gene family that balances cell proliferation and cell death. Development. 2020;147:dev184044-14. 


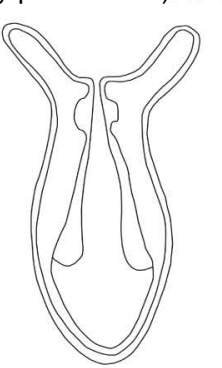

Uncut

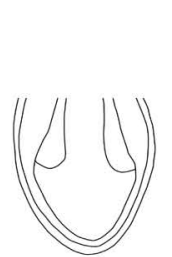

Time 0 cut

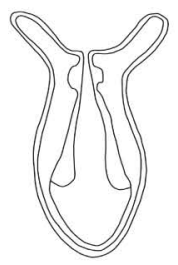

7dpa

aCC-BY-NC-ND 4.0 Interndif 6 al license.

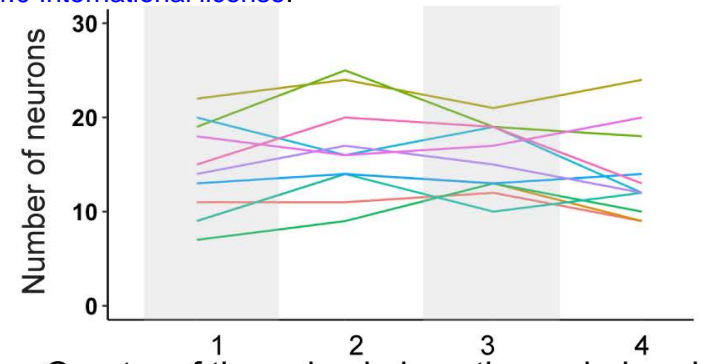

Quarter of the animal along the oral-aboral axis

\section{C}

D
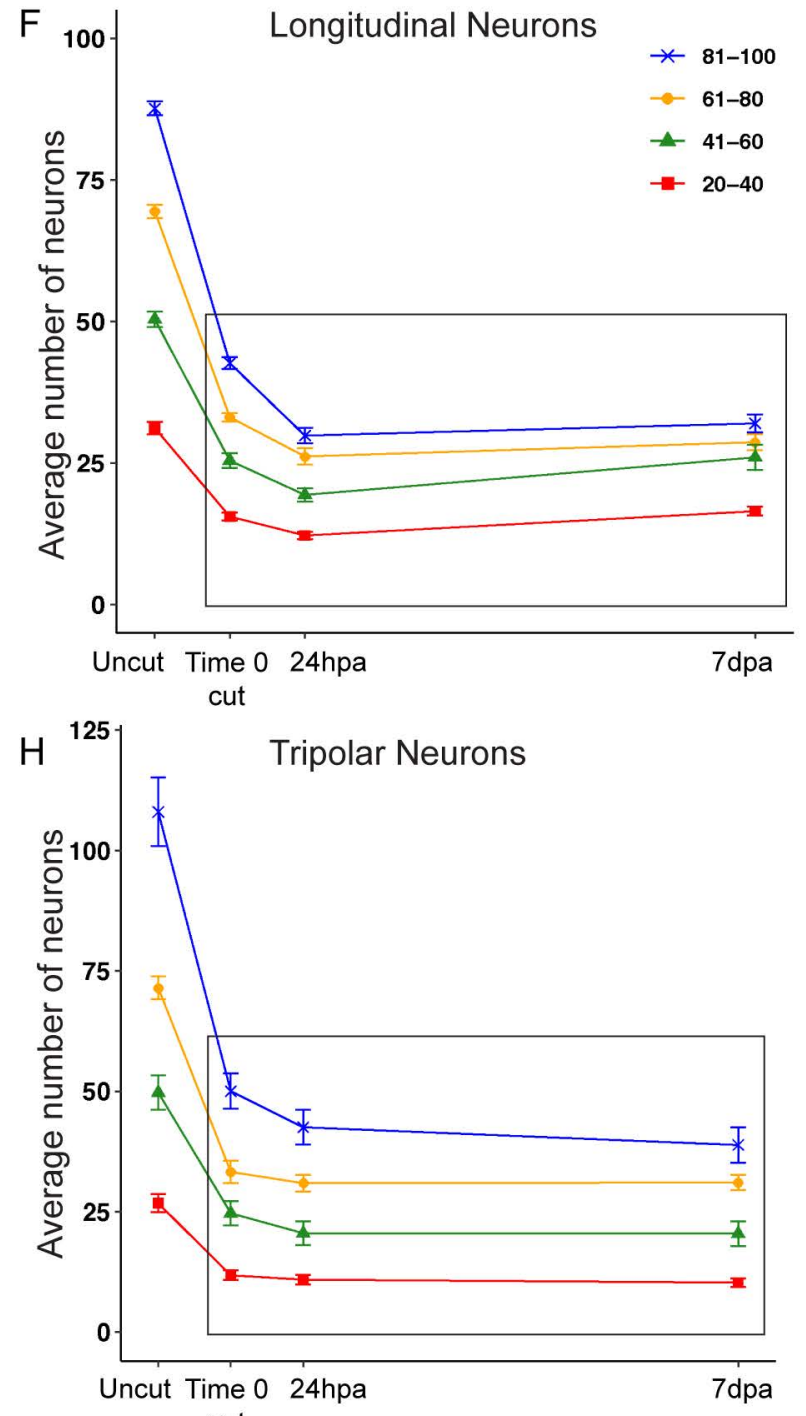

E
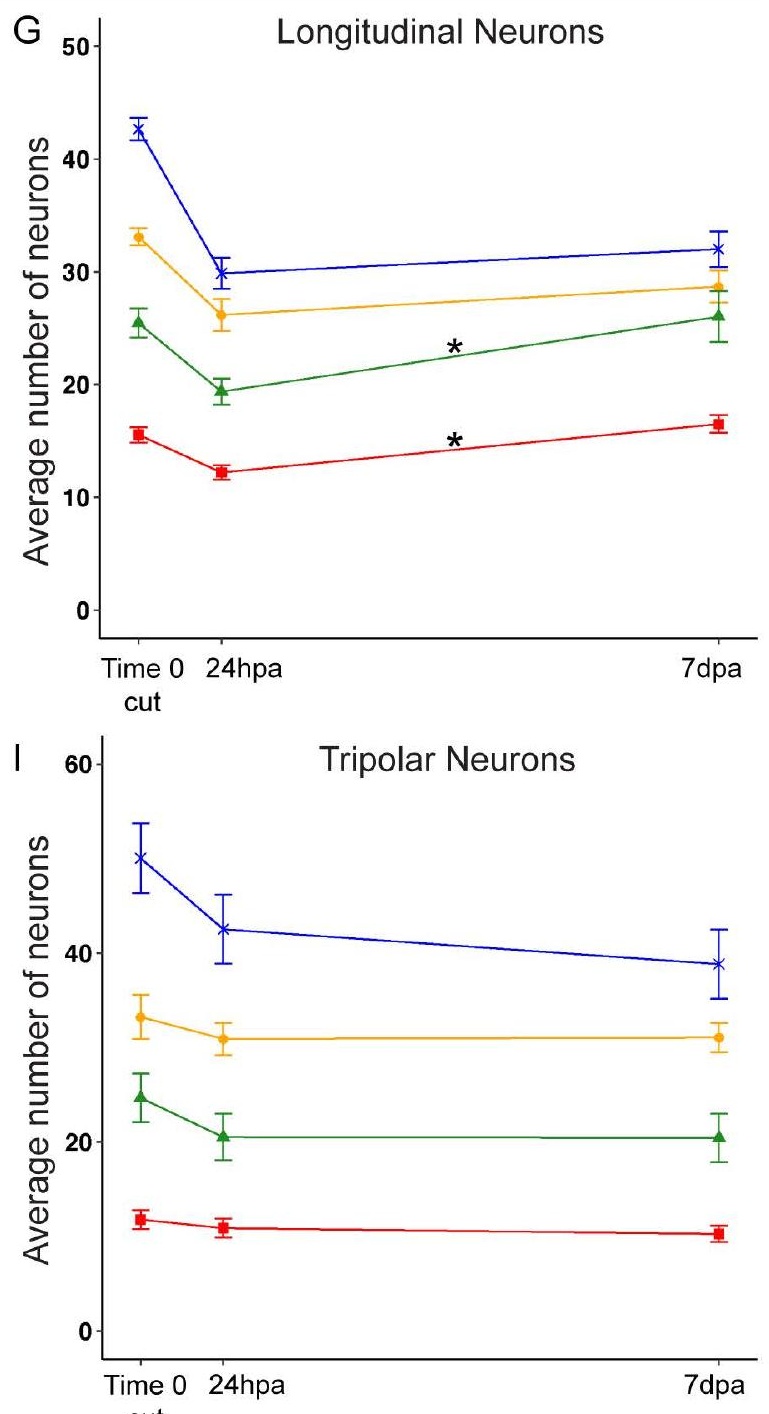


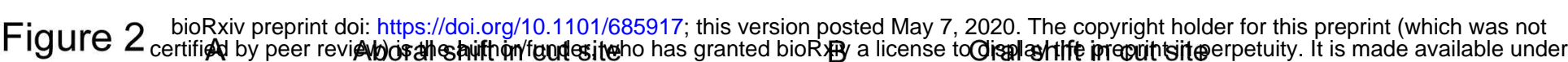
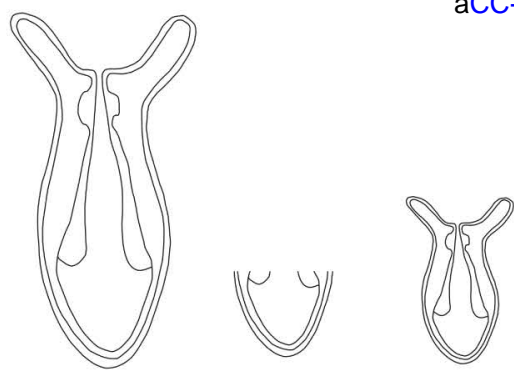

Uncut Time 0 cut

7dpa aCC-BY-NC-ND 4.0 International license.
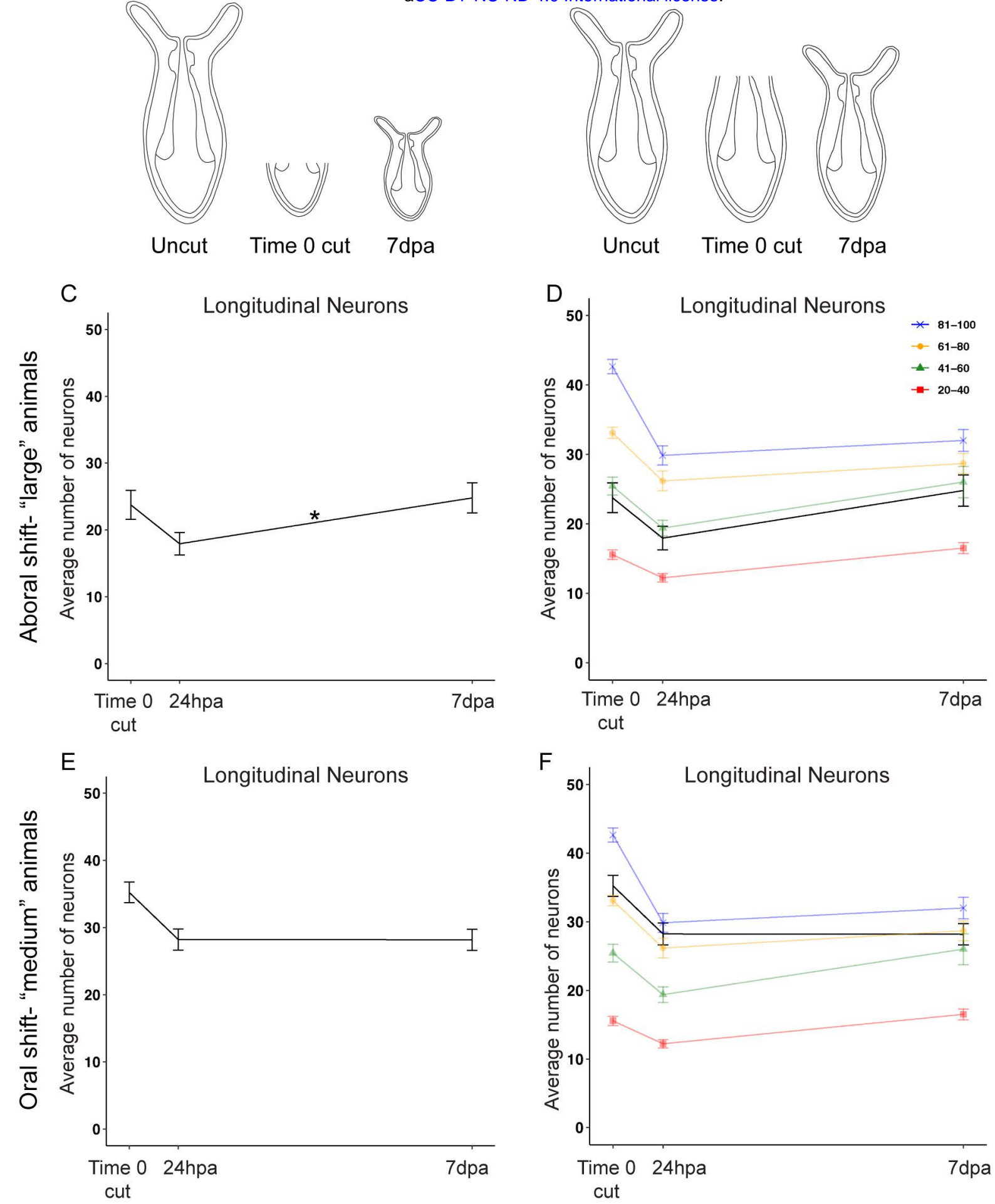

F
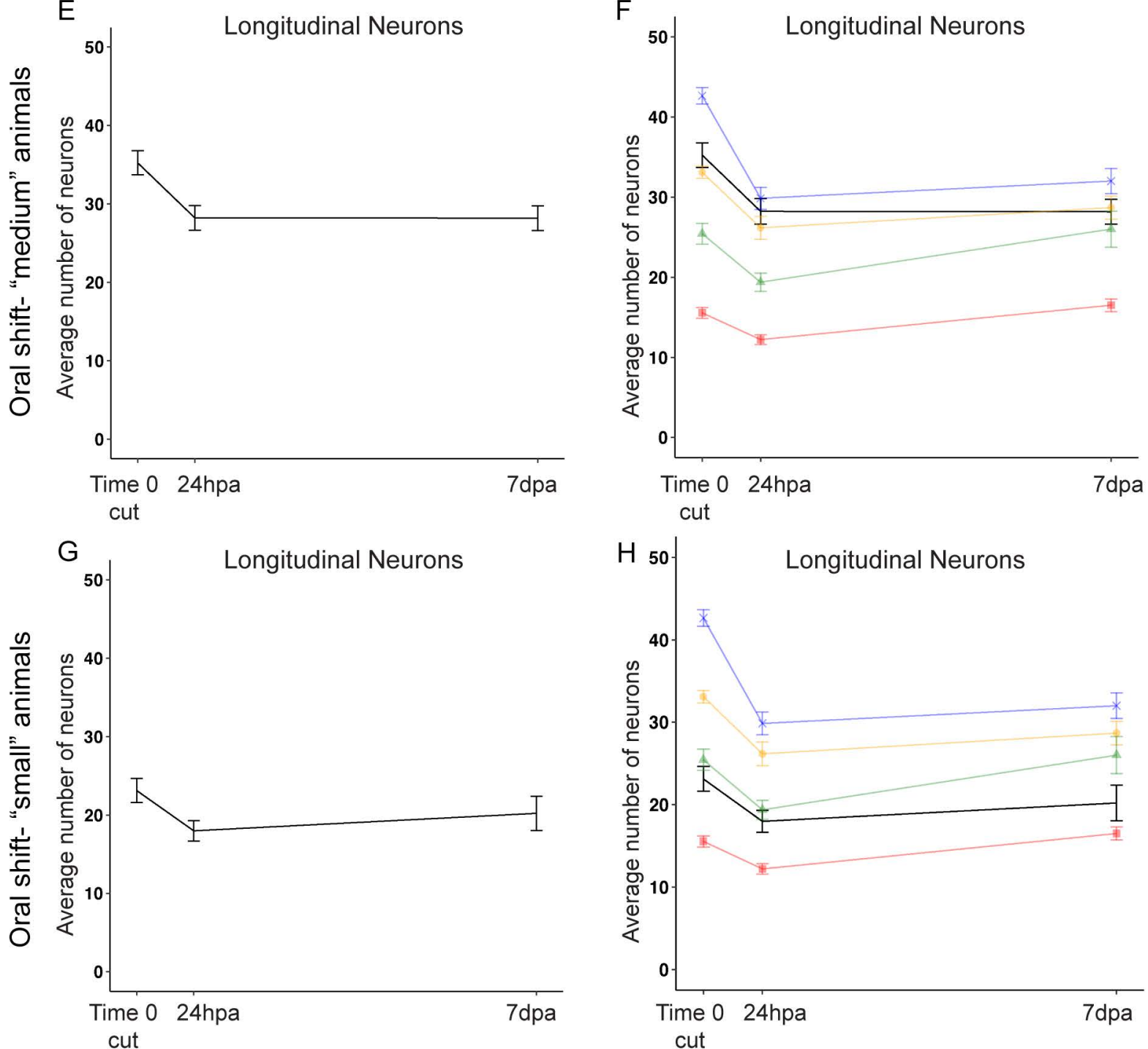

cut 
Week 1 Week 3 Week 7

Switched Feeding Regime

to display the preprint in perpetuity. It is made available under
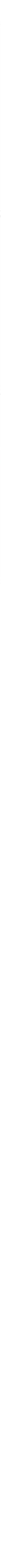

$E$

Tripolar Neurons

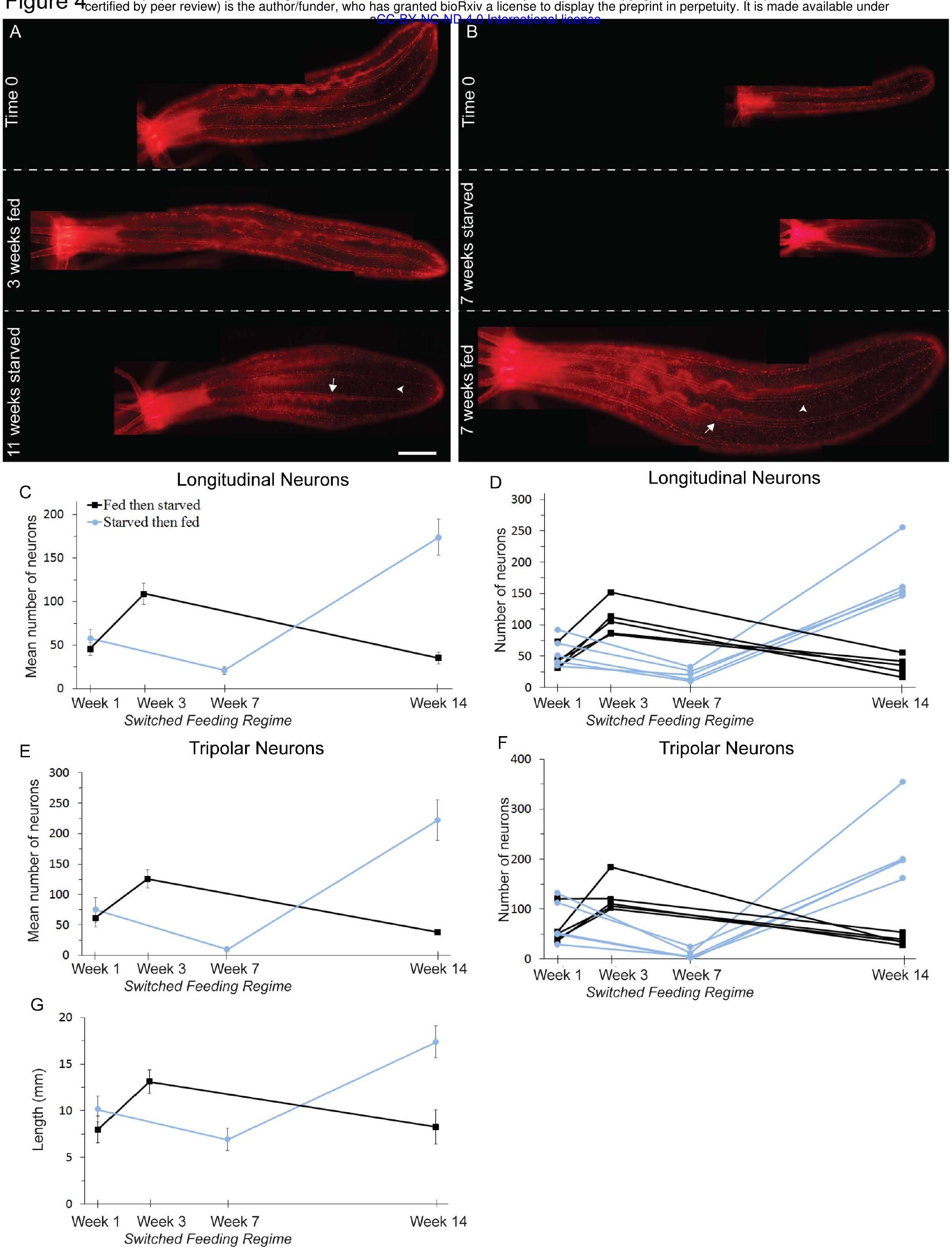

G
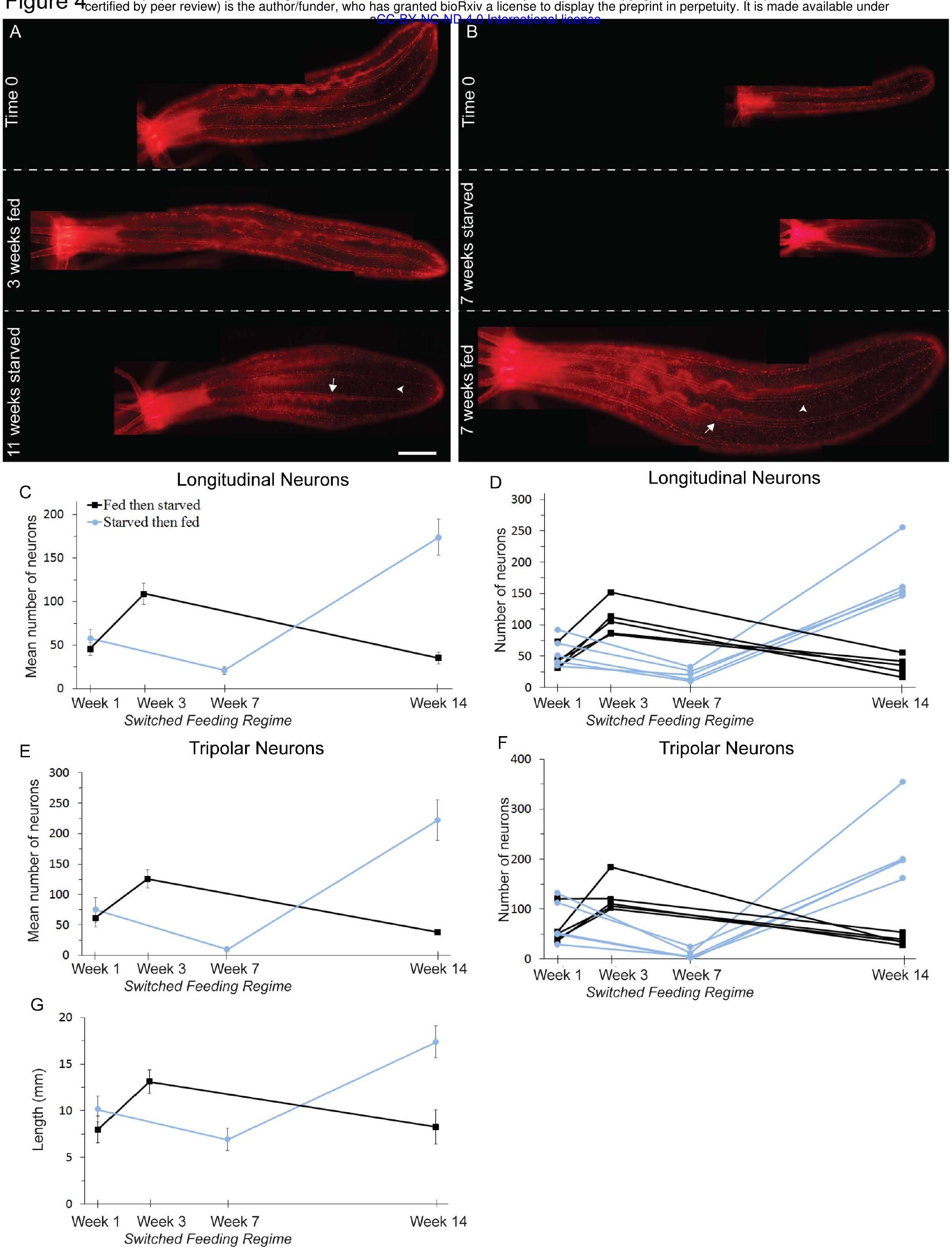

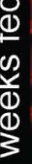

D

Switched Feeding Regime

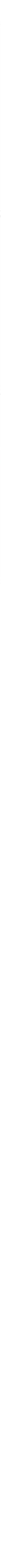


Supplemental Figure 1

A

7dpa
$A^{\prime}$

\section{7dpa}
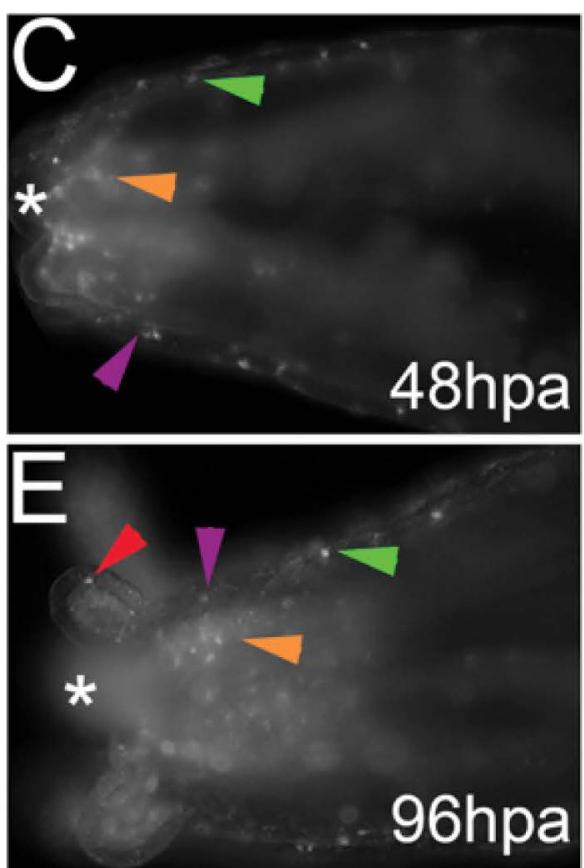

F

72hpa 


\section{Supplemental Figure 2}

A

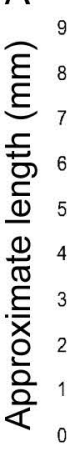

B

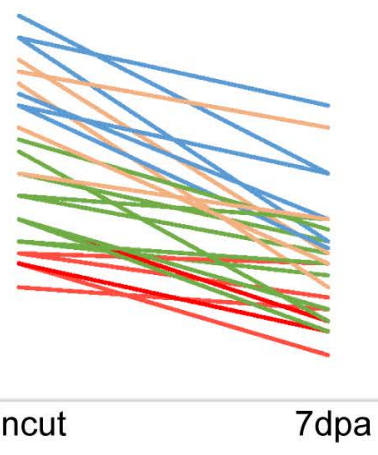

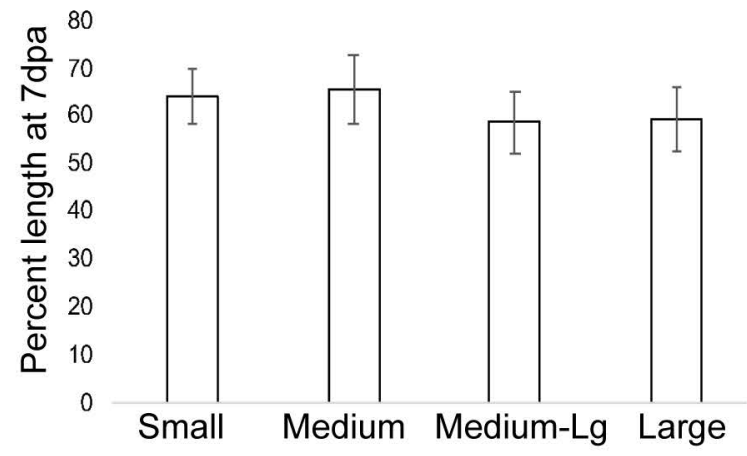

C

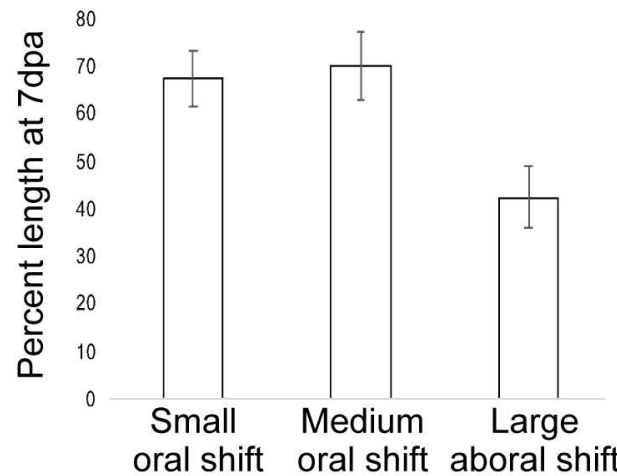

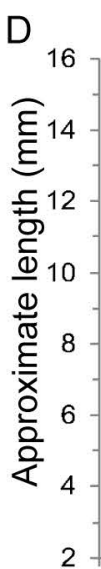
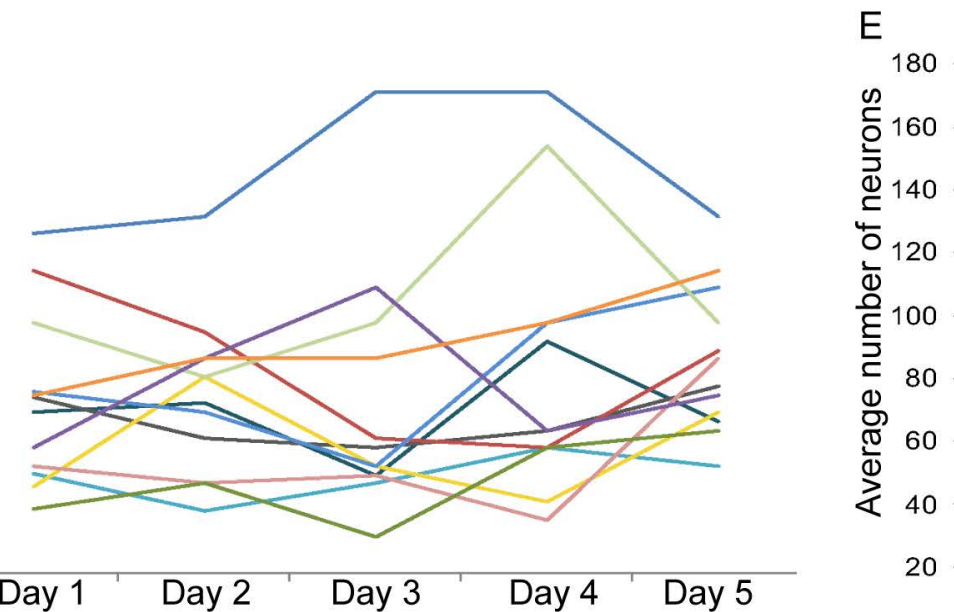

Longitudinal Neurons

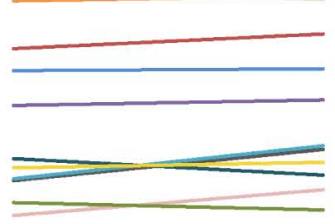

Day 1

Day 5 
A

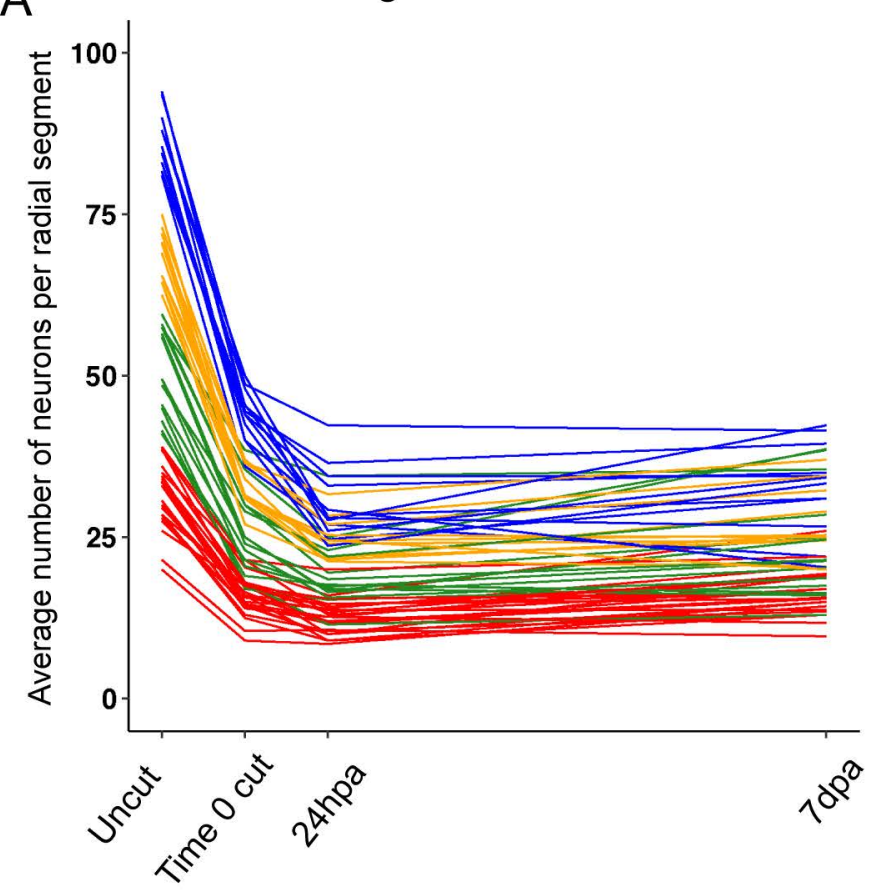

C

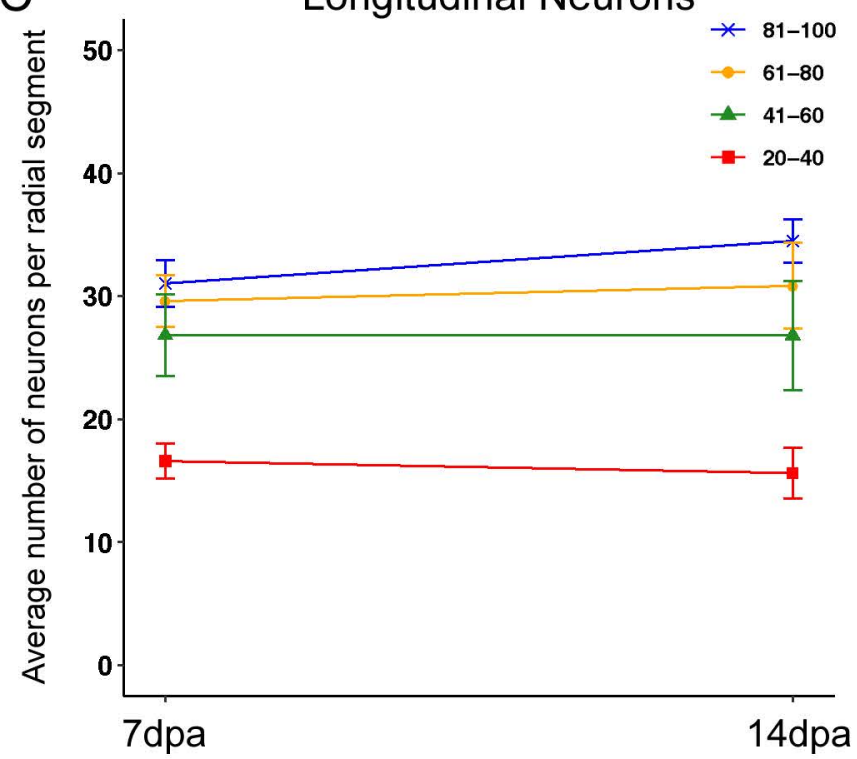

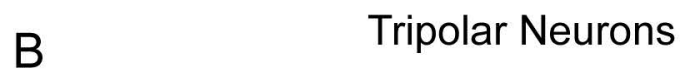

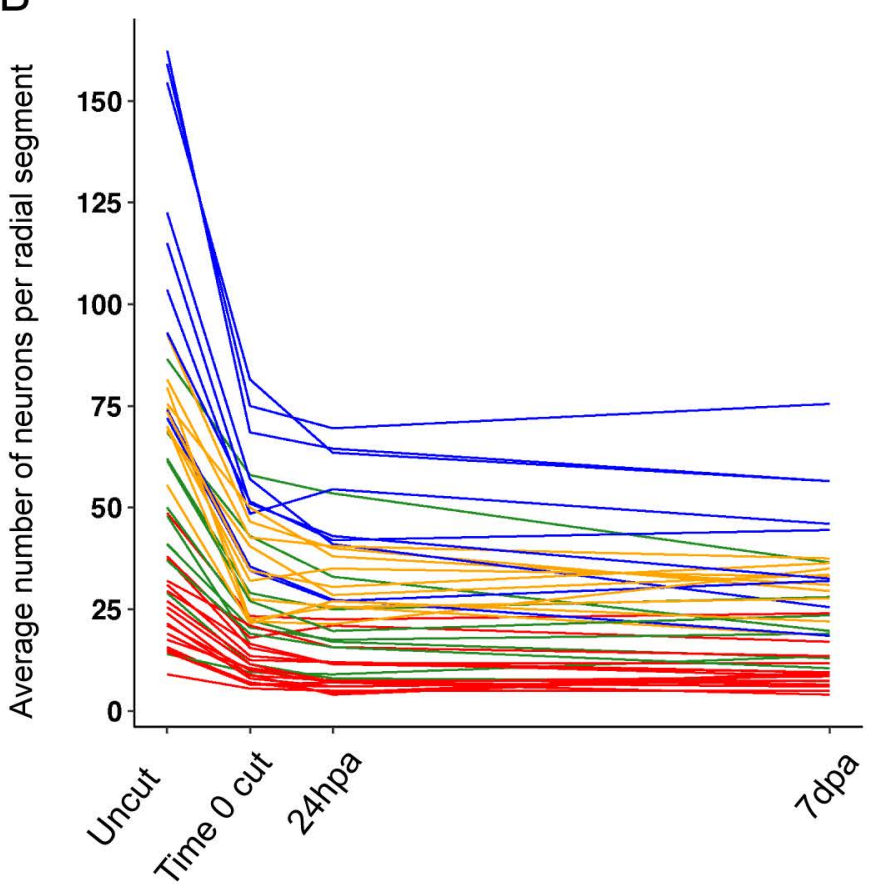

D

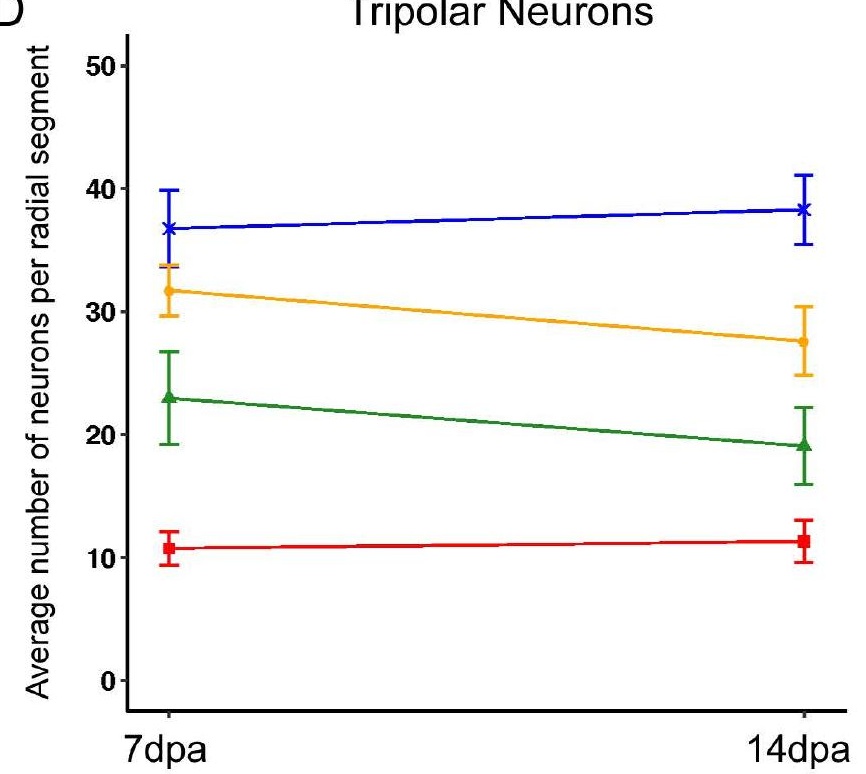




\section{Supplemental Figure 5}
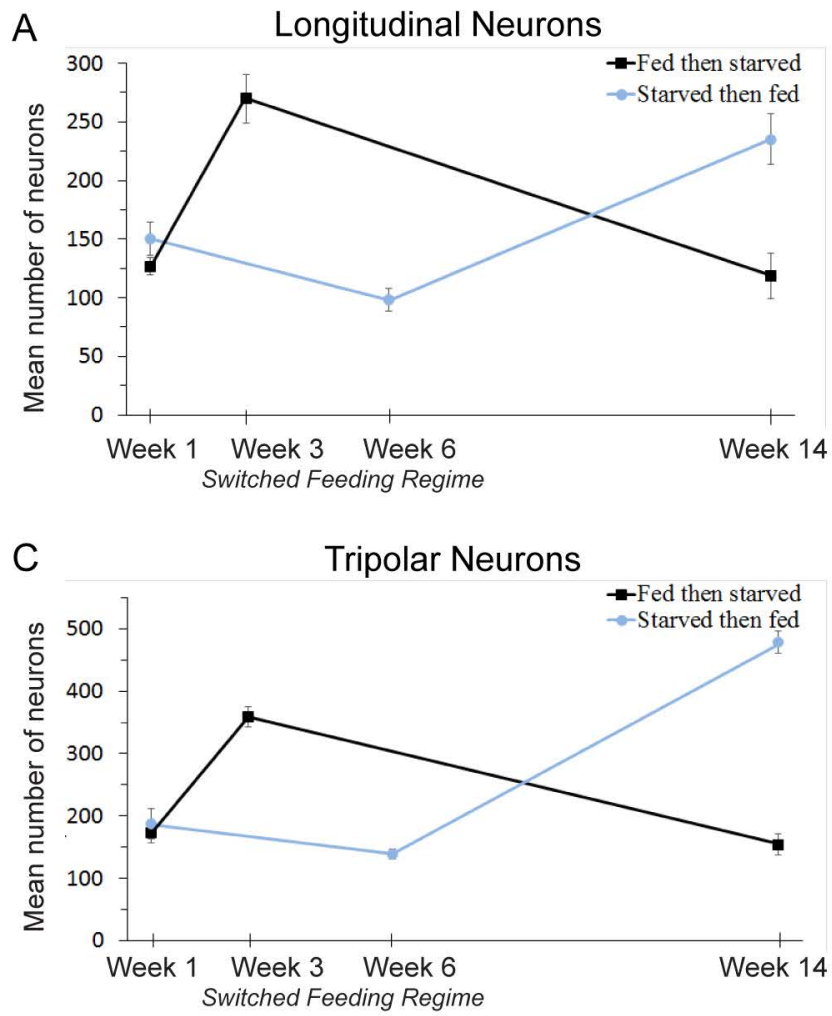

E

\subsection{Weeks Starved}

6
5
4
3
0

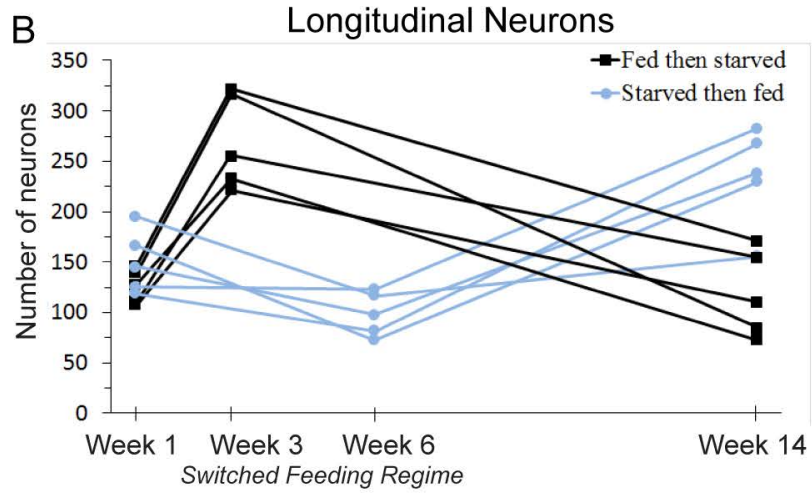

D

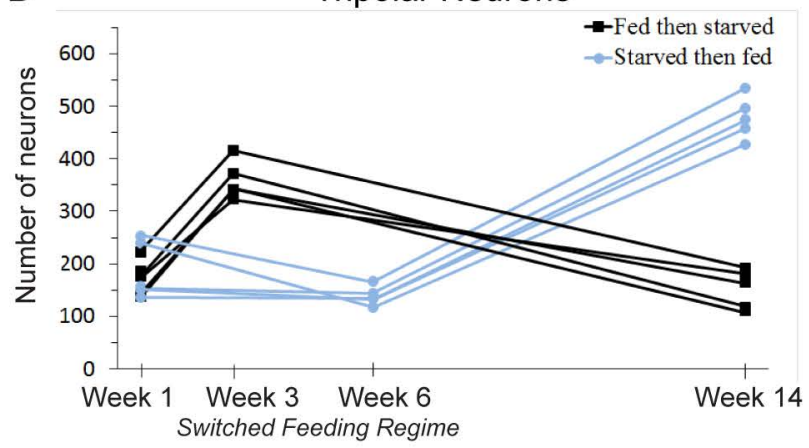

